\title{
THE HOMOLOGY OF CYCLIC PRODUCTS
}

\author{
BY \\ RICHARD G. SWAN $\left({ }^{1}\right)$
}

1. Introduction. A number of authors have considered the problem of determining the homology of cyclic products. The homology of all such products with rational coefficients was found by Richardson [17]. For $p$ a prime, the homology modulo $p$ of $p$-fold cyclic products was determined by Richardson and Smith [18]. More recently, Nakaoka [16] determined the cup products and Steenrod operations for this same case. The integral homology of 2 -fold cyclic products was found by S. K. Stein [23]. His method was extended to $p$-fold cyclic products for prime $p$ by Yoshioka [27]. The method does not give induced maps or cup products.

I will present here a method for calculating the homology, with arbitrary coefficients, of $n$-fold cyclic products for all $n$, not merely for the case where $n$ is a prime. The construction used is natural up to homotopy and so permits the determination of the map of this homology induced by any map of the original space. In particular, if we have a diagonal chain map for the original space, we can find one for the cyclic product and so can compute cup products.

In applying this method, it is not necessary to assume that the spaces involved are finite complexes. In fact, the method applies to any semisimplicial complex, finite or not.

In the case where the ground ring is a field, I will give an explicit natural formula for the cohomology ring of the cyclic product in terms of the cohomology ring of the original space (see $\$ 21$ ).

Throughout this paper, the symbol " $K$ " will denote the ground ring which I will assume to be either the integers $Z$, or a field. Many of the results will be seen to hold over much more general rings. A notable exception, however, is Lemma 16.2 on which the proof of the fundamental Theorems 6.2 and 12.1 is based. The method, with $K=Z$, yields a free chain complex having the homology of the cyclic product. By tensoring this complex with any abelian group or ring, we can find the homology with arbitrary coefficients as well as cup products and Bockstein operations with arbitrary coefficients.

The method is based on the theory of universal maps. Since this theory has not previously been developed in the context of categories and functors, I will devote $\$ 2$ to an exposition of this theory.

The method used here is a generalization of that used in my Princeton Doctoral Thesis (May, 1957) to compute the homology of $n$-fold cyclic products for prime $n$. The present results were announced in [25].

Received by the editors February 13, 1959.

(1) This work was done while the author was a National Science Foundation Fellow. 
I would like to thank P. E. Conner, S. MacLane, J. C. Moore, and N. E. Steenrod for their advice and encouragement in connection with this work. I would also like to thank the National Science Foundation for their support of this work.

2. Universal maps. In this section, I will develop the theory of universal maps $[1 ; 19]$ in the context of categories and functors.

Let $Q$ and $B$ be categories. I will denote by $M\left(A, A^{\prime}\right)$ the set of maps $f: A \rightarrow A^{\prime}$ in $Q$ and similarly for $B$. Suppose that for each pair of objects $A$ in $Q$ and $B$ in $B$ we are given a set $M(A, B)$. I will call $M(A, B)$ the set of a $B$-maps from $A$ to $B$ and write $f: A \rightarrow B$ instead of $f \in M(A, B)$. Suppose also that for every $A, A^{\prime} \in Q$ and $B, B^{\prime} \in B$, we are given functions

$$
\begin{aligned}
& M\left(A^{\prime}, B\right) \times M\left(A, A^{\prime}\right) \rightarrow M(A, B), \\
& M\left(B^{\prime}, B\right) \times M\left(A, B^{\prime}\right) \rightarrow M(A, B) .
\end{aligned}
$$

I will refer to these as compositions and write them in the usual way. They will be assumed to satisfy the following two conditions.

(a) Composition is associative. In other words, if $\alpha, \alpha^{\prime}$ are maps in $\alpha$, $\beta, \beta^{\prime}$ are maps in $\beta$, and $f$ is an $Q \beta$-map, then $(\beta f) \alpha=\beta(f \alpha),\left(\beta^{\prime} \beta\right) f=\beta^{\prime}(\beta f)$, and $(f \alpha) \alpha^{\prime}=f\left(\alpha \alpha^{\prime}\right)$ whenever these compositions are defined.

(b) If $f: A \rightarrow B$, then $f i_{A}=f$ and $i_{B} f=f$ where $i_{A}$ and $i_{B}$ are the identity maps of $A$ and $B$.

We can now define universal maps.

Definition 2.1. An $a ß$-map $u: A \rightarrow B$ is called right universal if, whenever $f: A \rightarrow B^{\prime}$ is an $Q B$-map with domain $A$, there is a unique map $g: B \rightarrow B^{\prime}$ in $B$ such that $f=g u$.

Dually, we say $u$ is left universal if, whenever $f: A^{\prime} \rightarrow B$ is an $Q ß$-map with range $B$, there is a unique map $g: A^{\prime} \rightarrow A$ in $a$ such that $f=u g$.

I will now show how to define a functor in terms of universal maps. Assume that for each object $A \in Q$, there is a right universal $Q B$-map $u: A \rightarrow B$. For each $A \in Q$, choose some particular right universal $Q$ Q $B$-map $u_{A}: A \rightarrow B$ and define $T(A)=B$. If $\alpha: A \rightarrow A^{\prime}$ in $\alpha$, define $T(\alpha): T(A) \rightarrow T\left(A^{\prime}\right)$ to be the unique map in $B$ such that $T(\alpha) u_{A}=u_{A^{\prime}} \alpha$. Because of the uniqueness condition in the definition of universal maps, we see immediately that $T$ is a covariant functor from $Q$ to $\beta$. The collection of chosen universal maps $u_{A}$ may now be regarded as a natural transformation from the identity functor to $T$.

Suppose now that we make different choices $u_{A}^{\prime}: A \rightarrow T^{\prime}(A)$ for the universal maps. This gives us a new functor $T^{\prime}$. For each $A \in \mathbb{Q}$, define $\eta_{A}: T(A) \rightarrow T^{\prime}(A)$ to be the unique map such that $\eta_{A} u_{A}=u_{A}^{\prime}$. In the same way, define $\eta_{A}^{\prime}: T^{\prime}(A) \rightarrow T(A)$. The uniqueness property now implies that $\eta_{A}$ and $\eta_{A}^{\prime}$ are natural transformations and are inverses. Consequently, $T$ and $T^{\prime}$ are naturally equivalent. In fact, the natural equivalences are canonical (i.e. uniquely defined). 
We can easily dualize the above construction by assuming that each object $B \in B$ has a left universal $Q B$-map $u_{B}: T(B) \rightarrow B$. This defines a functor $T: B \rightarrow Q$. Note that $T$ is still covariant.

The reader will easily supply the proper categories $Q$ and $B$ for each of the examples in [1] and [19]. The direct sum and direct limit functors can easily be characterized by means of right universal maps, while the direct product and inverse limit functors are characterized by left universal maps.

The theory of adjoint functors [13] may also be developed within the framework of the theory of universal maps. Let $Q$ and $B$ be categories and $S: B \rightarrow Q$ a functor. Define the set $M(A, B)$ of $Q \beta$-maps by $M(A, B)$ $=M(A, S(B))$ with the obvious compositions. Suppose every object $A \in Q$ has a right universal Qß-map $u_{A}: A \rightarrow T(A)$. Define $\phi: M(T(A), B)$ $\rightarrow M(A, S(B))$ by $\phi(f)=f u_{A}$. It then follows immediately from the definition of universal maps that $\phi$ is a natural isomorphism. Consequently, $T$ is an adjoint functor of $S$. Conversely, let $T$ be any adjoint functor of $S$. This means there is a natural isomorphism

$$
\phi: M(T(A), B) \rightarrow M(A, S(B))=M(A, B) .
$$

Define an $Q ß$-map $u_{A}: A \rightarrow T(A)$ by $u_{A}=\phi\left(i_{T(A)}\right)$ where $i_{T(A)}$ is the identity map of $T(A)$. Then, for all $f \in M(T(A), B), \phi(f)=f u_{A}$. Since $\phi$ is an isomorphism, it follows that $u_{A}$ is right universal. The uniqueness of adjoint functors [13] now follows immediately from the uniqueness of functors defined by universal maps.

By dualizing the above construction, we can define $S$ starting with $T$.

An example is given by taking $Q$ to be the category of semisimplicial complexes, $B$ the category of topological spaces, and $S$ the functor which associates to each space its singular complex. In this case, $T(A)$ is the geometric realiziation of $A$ as defined by Milnor [14].

The generalization to functors of more than one variable is left to the reader. For example, the relation $\operatorname{Hom}(A, \operatorname{Hom}(B, C))=\operatorname{Hom}(A \otimes B, C)$ leads to a characterization of Hom by means of left universal maps.

3. H-categories. This section deals with categories in which there is defined a relation with the usual properties of homotopy.

Definition 3.1. An $H$-category is a category $a$ together with an equivaIence relation $\simeq$ called "homotopy" on each of the sets $M\left(A, A^{\prime}\right)$ such that $\simeq$ is a congruence relation in $a$. In other words, if $f \simeq f^{\prime}$ and $g \simeq g^{\prime}$ then $f g \simeq f^{\prime} g^{\prime}$ provided $f g$ is defined.

REMARK 3.1. This notion should not be confused with the notion of an $h$-category [11, Chapter IV, §9].

REMARK 3.2. It is easy to generalize this concept by requiring composition in $Q$ to be associative up to homotopy but not necessarily strictly associative. We may also assume that the identity maps in $a$ are identities only up to homotopy.

If $Q$ is an $H$-category, we can define a quotient category $Q^{*}$ which has the 
same objects as $Q$ but whose maps are the homotopy classes of maps in $Q$. The identification map $J: Q \rightarrow Q^{*}$ is obviously a covariant functor.

Definition 3.2. Let $Q$ be any category and let $\beta$ be an $H$-category. A function $T: Q \rightarrow B$ is called a covariant (contravariant) prefunctor if the composition $J T$ is a covariant (contravariant) functor. Here, $J$ is the quotient map just defined. We say that $T$ is a representative of the functor $J T$.

REMARK 3.3. Clearly, $T$ is a covariant prefunctor if and only if it sends objects into objects, maps into maps, preserves range and domain, and satisfies

(a) $T\left(i_{A}\right) \simeq i_{T(A)}$ for all objects $A \in Q$,

(b) $T(f g) \simeq T(f) T(g)$ whenever $f g$ is defined.

Definition 3.3. Let $Q$ and $\beta$ be as in Definition 3.2. Let $S$ and $T$ be covariant prefunctors from $a$ to $B$. A natural transformation $\eta: S \rightarrow T$ is defined to be a collection of maps $\eta_{A}: S(A) \rightarrow T(A)$, one for each object $A \in Q$ such that, if $f: A \rightarrow A^{\prime}$ in $Q$ then $T(f) \eta_{A} \simeq \eta_{A^{\prime}} S(f)$. A natural transformation of contravariant prefunctors is defined similarly.

REMARK 3.4. Each such natural transformation $\eta$ determines a natural transformation of functors $\eta_{*}: J S \rightarrow J T$. Conversely, $\eta_{*}$ determines $\eta$ up to homotopy.

Definition 3.4. A natural transformation $\eta: S \rightarrow T$ of prefunctors is called a natural equivalence of prefunctors if there is another natural transformation $\xi: T \rightarrow S$ such that $\eta_{A} \xi_{A} \simeq i_{T(A)}$ and $\xi_{A} \eta_{A} \simeq i_{S(A)}$ for all $A \in Q$.

REMARK 3.5. If $\eta$ is a natural equivalence of prefunctors, then $\eta_{*}: J S \rightarrow J T$ is a natural equivalence of functors.

Definition 3.5. Let $Q$ and $B$ be $H$-categories. A prefunctor $T: Q \rightarrow B$ is said to preserve homotopy if $f \simeq g$ in $Q$ implies $T(f) \simeq T(g)$ in $B$.

REMARK 3.6. This condition is clearly equivalent to the existence of a functor $T_{*}: Q^{*} \rightarrow B^{*}$ such that $J T=T_{*} J$. Obviously, $T_{*}$ is uniquely determined by $T$.

I will now give a simple example to illustrate these concepts. Let $I$ be the $H$-category of triangulable (but not triangulated) pairs $(X, A)$ with the usual maps and homotopies. Let $\mathrm{C}$ be the $H$-category of chain complexes (over some ground ring $K$ ) with chain maps and chain homotopies. Define a covariant prefunctor $C: \mathfrak{I} \rightarrow \mathfrak{C}$ as follows.

For each $(X, A)$ in $J$, choose some triangulation and let $C(X, A)$ be the chain complex of the resulting simplicial pair. For each map $f:(X, A)$ $\rightarrow(Y, B)$ in $\mathrm{J}$, choose an "algebraic approximation" $C(f): C(X, A) \rightarrow C(Y, B)$. It then follows from well known theorems that $C$ is a covariant prefunctor which preserves homotopy.

Suppose now that we choose different triangulations and algebraic approximations, getting a new prefunctor $C^{\prime}$. For each $(X, A)$ in $J$, choose a chain map $\eta_{(X, A)}: C(X, A) \rightarrow C^{\prime}(X, A)$ which is an algebraic approximation to the identity map of $(X, A)$. Then it is easy to see that $\eta$ is a natural equiva- 
lence of prefunctors. Therefore, any two choices for $C$ are naturally equivalent. As a matter of fact, the natural equivalence $\eta$ is canonical in the sense that it is unique up to homotopy.

4. $h$-universal maps. The theory of universal maps may also be modified by considering maps which are universal up to homotopy. Suppose $Q$ and $B$ are $H$-categories. Suppose also that for each object $A$ in $Q$ and object $B$ in $B$ we are given a set $M(A, B)$ and composition functions as in $\S 2$. Suppose also that we are given an equivalence relation $\simeq$, called homotopy, on each of the sets $M(A, B)$. The compositions will not be assumed to satisfy conditions (a) and (b) of $\$ 2$ but only the following three conditions.

(a) Composition is associative up to homotopy i.e. if $\alpha, \alpha^{\prime} \in Q, \beta, \beta^{\prime} \in ß$ and $f$ is an $Q\left(\beta\right.$-map, then $(\beta f) \alpha \simeq \beta(f \alpha),\left(\beta^{\prime} \beta\right) f \simeq \beta^{\prime}(\beta f),(f \alpha) \alpha^{\prime} \simeq f\left(\alpha \alpha^{\prime}\right)$ provided these compositions are defined.

(b) If $f: A \rightarrow B$ is an $Q \beta$-map, then $f i_{A} \simeq f$ and $i_{B} f \simeq f$.

(c) If $\alpha \simeq \alpha^{\prime}, \beta \simeq \beta^{\prime}, f \simeq f^{\prime}$, then $\beta f \alpha \simeq \beta^{\prime} f^{\prime} \alpha^{\prime}$.

Definition 4.1. An $Q B$-map $u: A \rightarrow B$ is right $h$-universal if, whenever $f: A \rightarrow B^{\prime}$ is an QB-map with domain $A$, there is a map $\beta$ in $B$ satisfying $f \simeq \beta u$, and any two such maps $\beta$ are homotopic.

Left $h$-universal maps are defined dually.

The construction of the functor $T$ given in $\$ 2$ may now be repeated provided every object $A$ in $Q$ has a right $h$-universal map $u: A \rightarrow T(A)$. However, we can only assert that $T$ is a prefunctor. If we make different choices for the universal maps, there will be a natural equivalence $\eta$ between the resulting prefunctors. This $\eta$ is canonical in the sense that it is unique up to homotopy.

An obvious example of a right $h$-universal map is given by taking $a$ to be an exact category [2, Appendix], letting $\Theta$ be the $H$-category of injective right complexes in $Q$, and defining an $Q B$-map to be an augmentation. An injective resolution of $A$ is clearly a right $h$-universal map. The method I will use to find the homology of cyclic products is a generalization of this example.

Similarly, we can get a left $h$-universal map by taking $B$ to be the exact category and letting $Q$ be the $H$-category of left projective complexes in $B$. A projective resolution is then left $h$-universal.

An interesting example of left $h$-universal maps was suggested by J. W. Milnor. Let $\mathcal{G}$ be a category of topological groups and continuous homomorphisms. We consider only trivial homotopies (i.e. equalities) in g. Let $x$ be an $H$-category of topological spaces with the usual maps and homotopies. For $X$ in $x$ and $G$ in $\mathcal{G}$, let $M(X, G)$ be the collection of principal fiber bundles with base $X$ and group $G$. A "homotopy" in $M(X, G)$ will be an equivalence of bundles. Compositions are defined by taking induced bundles. A left $h$-universal map $u: X \rightarrow G$ is just a universal bundle for $G$. Therefore, $T(G)$ will be a classifying space for $G$.

In the application of the theory to cyclic products, we will encounter $h$ universal maps with an additional property. 
Definition 4.2. A right $h$-universal $Q B$-map $u: A \rightarrow B$ is called special if, whenever $f: A \rightarrow B^{\prime}$ is an $Q B$-map with domain $A$, there is a map $\beta: B \rightarrow B^{\prime}$ in $B$ such that $f=\beta u$ (not just $f \simeq \beta u$ ).

Special left $h$-universal maps are defined dually.

Note that $\beta$ is not assumed unique. It is, of course, unique up to homotopy.

In defining the prefunctor $T$, we will naturally choose special $h$-universal maps $u_{A}: A \rightarrow T(A)$ whenever possible and choose $T(\alpha)$ so that $T(\alpha) u_{A}=u_{A^{\prime}} \alpha$. Note that this does not necessarily make $T$ a functor since $T(\alpha) T\left(\alpha^{\prime}\right) u_{A}$ $=T\left(\alpha \alpha^{\prime}\right) u_{A}$ only implies $T(\alpha) T\left(\alpha^{\prime}\right) \simeq T\left(\alpha \alpha^{\prime}\right)$.

5. Cyclic products. Let $X$ be a semisimplicial complex [15]. Define $X^{n}$ to be the $n$-fold Cartesian product of $X$ with itself. Let $\pi$ be a cyclic group of order $n$ with generator $T$. This group acts on $X^{n}$ by cyclic permutation

$$
T\left(x_{1}, \cdots, x_{n}\right)=\left(x_{n}, x_{1}, \cdots, x_{n-1}\right) .
$$

The $n$-fold cyclic product of $X$ is defined by

$$
C P^{n} X=X^{n} / \pi \text {. }
$$

In other words, $C P^{n} X$ is the quotient complex of $X^{n}$ obtained by making the identifications $x \sim T x$ for all $x \in X^{n}$.

If $A \subset X$ is a subcomplex of $X$, define $(X, A)^{n}$ to be $\left(X^{n}, A_{0}\right)$ where $A_{0}$ is the set of $\left(x_{1}, \cdots, x_{n}\right)$ with at least one $x_{i} \in A$. In this case, $C P^{n}(X, A)$ is defined to be $\left(X^{n} / \pi, A_{0} / \pi\right)$. I will state all results for the absolute case only, the corresponding results in the relative case being exactly the same.

Dold [6] has shown that the homology of $C P^{n} X$ is determined by the homology of $X$. His method, however, is not suitable for actually calculating this homology.

If $X$ is any semisimplicial complex, $C(X)$ will denote the normalized chain complex of $X$ [15, Chapter III]. Obviously, $C\left(C P^{n} X\right)=C\left(X^{n}\right) / \pi$. If $A$ is any $K$-module, $\otimes^{n} A$ will denote the $n$-fold tensor product of $A$ with itself. The group $\pi$ acts on this by

$$
T\left(a_{1} \otimes \cdots \otimes a_{n}\right)=(-1)^{\epsilon} a_{n} \otimes a_{1} \otimes \cdots \otimes a_{n-1}
$$

where $\epsilon=\left(\operatorname{dim} a_{n}\right)\left(\operatorname{dim} a_{1}+\cdots+a_{n-1}\right)$.

The Eilenberg-Zilber map [10, Chapter II, $2 ; 15$, Chapter III]

$$
\nabla: \otimes^{n} C(X) \rightarrow C\left(X^{n}\right)
$$

is a $\pi$-equivariant homotopy equivalence.

If $x$ is any simplex of $X$, there is a unique nondegenerate simplex $y$ of $X$ such that $x$ is obtained by applying degeneracy operators to $y$. I will define the reduced dimension of $x$ by $d_{r}(x)=\operatorname{dim} y$. The $k$-skeleton $S k_{k} X$ is the set of all $x$ in $X$ with $d_{r}(x) \leqq k$. It is clearly a subcomplex. 
Define a filtration of $X^{n}$ by letting $\Phi_{k} X^{n}$ be the set of $\left(x_{1}, \cdots, x_{n}\right)$ such that $\sum d_{r}\left(x_{i}\right) \leqq k$. This is obviously a subcomplex of $X^{n}$ so there is an induced filtration of chains $\Phi_{k} C\left(X^{n}\right)$. The definition of the Eilenberg-Zilber map shows that $\nabla$ maps the $k$-dimensional part of $\otimes^{n} C(X)$ into $\Phi_{k} C\left(X^{n}\right)$.

The basic property of this filtration is given by the following lemma.

Lemma 5.1. Let $\pi^{\prime}$ be the subgroup of $\pi$ of order $r$. Let $e$ be a nondegenerate simplex of $\Phi_{k} X^{n}$ which is fixed under $\pi^{\prime}$. Then $\operatorname{dim} e \leqq k / r$.

Proof. If $e$ is fixed under $\pi^{\prime}$ it must have the form

$$
\left(x_{1}, \cdots, x_{n / r}, x_{1}, \cdots, x_{n / r}, \cdots, x_{1}, \cdots, x_{n / r}\right) .
$$

Since $e$ is in $\Phi_{k}$, we have $r \sum d_{r}\left(x_{i}\right) \leqq k$. Since $e$ is nondegenerate, so is the simplex $\left(x_{1}, \cdots, x_{n / r}\right)$ in $X^{n / r}$ and so $\operatorname{dim}\left(x_{1}, \cdots, x_{n / r}\right)=d_{r}\left(x_{1}, \cdots, x_{n / r}\right)$. The required result now follows from the next lemma.

Lemma 5.2. Let $X_{1}, \cdots, X_{h}$ be semisimplicial complexes. Let $\left(x_{1}, \cdots, x_{h}\right)$ be a simplex of $X_{1} \times \cdots \times X_{h}$. Then $d_{r}\left(x_{1}, \cdots, x_{h}\right) \leqq \sum d_{r}\left(x_{i}\right)$.

Proof. It is sufficient to prove this for $h=2$ and then use induction. Let $x_{1}=s_{\nu_{q}} \cdots s_{\nu_{1}} y_{1}$ and $x_{2}=s_{\mu_{p}} \cdots s_{\mu_{1}} y_{2}$ with $y_{1}$ and $y_{2}$ nondegenerate and $\nu_{q}>\cdots>\nu_{1}, \mu_{p}>\cdots>\mu_{1}$. It is easy to reduce any sequence $s_{i_{q}} \cdots s_{i_{1}}$ to this form. We merely choose the representative for which $i_{1}+\cdots+i_{q}$ is maximal. Now, if some $\nu_{i}=\mu_{j}$, we can bring $s_{\nu_{i}}$ and $s_{\mu_{j}}$ to the left of both expressions getting $x_{1}=s_{v_{i}} z_{1}$ and $x_{2}=s_{\mu_{j}} z_{2}$. Thus $\left(x_{1}, x_{2}\right)=s_{v_{i}}\left(z_{1}, z_{2}\right)$. The result will then follow by induction if it has been proved for elements of dimension $<\max \operatorname{dim} x_{i}$.

Suppose now that $s_{\nu_{i}} \neq s_{\mu_{j}}$ for all $i$ and $j$. Then $\operatorname{dim}\left(x_{1}, x_{2}\right)=q+\operatorname{dim} y_{1}$ $=p+\operatorname{dim} y_{2}=m$ say. Now, $\mu_{p} \leqq m-1$ and $\nu_{q} \leqq m-1$. All $\mu_{i}$ and $\nu_{j}$ are distinct and lie in the interval $[0, m]$. Therefore, $p+q \leqq m$. But,

$$
2 m=\left(q+\operatorname{dim} y_{1}\right)+\left(p+\operatorname{dim} y_{2}\right) \leqq m+\operatorname{dim} y_{1}+\operatorname{dim} y_{2} .
$$

Therefore,

$$
m \leqq \operatorname{dim} y_{1}+\operatorname{dim} y_{2}=d_{r}\left(x_{1}\right)+d_{r}\left(x_{2}\right) .
$$

6. The categories. The homology of $C P^{n}(X)$ will be found as follows. The map $\nabla$ factors through a certain "almost" $h$-universal map (see §12) $u: \otimes{ }^{n} C(X) \rightarrow F\left(\otimes^{n} C(X)\right)$. This gives $\nabla=g u$ where $g: F\left(\otimes{ }^{n} C(X)\right) \rightarrow C\left(X^{n}\right)$. We then consider

$$
g / \pi: F\left(\otimes^{n} C(X)\right) / \pi \rightarrow C\left(C P^{n} X\right)
$$

and show it induces an isomorphism of homology. For computation, we can replace $C(X)$ by any other $K$-free chain complex having the same homology.

In order to define the $h$-universal map $u$, we consider some special categories. 
Definition 6.1. An object of the category $\beta$ is a chain complex $P$ over $K \pi$ satisfying the following conditions.

(a) $P$ is free over $K$ and has a basis which is permuted by $\pi$ up to sign. In other words, $P$ has a $K$-base $\left\{e_{i}\right\}$ such that if $t \in \pi$, then $t e_{i}= \pm e_{j}$ for some $j$.

(b) If $e_{i}$ is a basis element and $t e_{i}= \pm e_{i}$ then $\operatorname{dim} e_{i}$ is a multiple of the order of $t$. If $t \neq 1$ and the order of $t$ is a power of 2, this multiple is even for $t e_{i}=e_{i}$ and odd for $t e_{i}=-e_{i}$.

The maps in $\beta$ are all $\pi$-equivariant chain maps. The only homotopies in $P$ will be the trivial ones (equalities).

Obviously, if $M$ is any $K$-free chain complex then $\otimes^{n} M$ is in $P$, the base being given by $e_{i_{1}} \otimes \cdots \otimes e_{i_{n}}$ where $\left\{e_{i}\right\}$ is a base for $M$.

Definition 6.2. An object of the category $\mathcal{F}$ is a filtered chain complex $F$ over $K \pi$. A map in $\mathcal{F}$ is a chain map over $K \pi$ which preserves filtration. A homotopy in $\mathcal{F}$ is a chain homotopy $S$ over $K \pi$ which preserves filtration, i.e. $S \Phi_{k}(F) \subset \Phi_{k}\left(F^{\prime}\right)$ not just $S \Phi_{k}(F) \subset \Phi_{k+1}\left(F^{\prime}\right)$. Obviously $C\left(X^{n}\right)$ is in $\mathcal{F}$, the filtration being that defined in $\$ 5$.

Definition 6.3. A PF-map $f: P \rightarrow F$ is a chain map over $K \pi$ such that $f\left(P_{k}\right) \subset \Phi_{k}(F)$ for all $k$. A PF-homotopy is a chain homotopy $S$ over $K \pi$ such that $S\left(P_{k}\right) \subset \Phi_{k}(F)$ for all $k$.

Note that we require $S\left(P_{k}\right) \subset \Phi_{k}(F)$ and not just $S\left(P_{k}\right) \subset \Phi_{k+1}(F)$.

Clearly the map $\nabla: \otimes{ }^{n} C(X) \rightarrow C\left(X^{n}\right)$ is a PF-map.

We cannot find a universal $\odot \mathcal{F}$-map for every $P$ in $P$. It is necessary to consider subcategories of $\mathcal{F}$.

For each prime $p$, let $\pi_{p}$ be the (unique) $p$-Sylow subgroup of $\pi$ and let $G_{p}$ be the (unique) complement of $\pi_{p}$ in $\pi$. $G_{p}$ is the direct sum of the $\pi_{q}$ for $q \neq p$.

Definition 6.4. Let $p$ be a prime. The category $\mathcal{F}_{p}$ is the full subcategory of $\mathcal{F}$ whose objects $F$ satisfy the following conditions.

(a) $F$ has a basis $\left\{e_{i}\right\}$ over $K$ which is permuted up to sign by $\pi$.

(b) Each $\Phi_{k}(F)$ is generated by some subset of this basis.

(c) If $t \in \pi_{p}$ and $t e_{i}= \pm e_{i}$, then $t e_{i}=+e_{i}$ (i.e. $t e_{i}=-e_{i}$ cannot occur).

(d) Let $\pi^{\prime} \subset \pi_{p}$ have order $r$. If $e_{i}$ is a basis element fixed under $\pi^{\prime}$ and $e_{i} \in \Phi_{k}(F)$ then $\operatorname{dim} e_{i} \leqq k / r$.

We let $\mathcal{F}_{\infty}$ be the intersection of all the categories $\mathcal{F}_{p}$. Lemma 5.1 implies that $C\left(X^{n}\right) \in \mathcal{F}_{\infty}$.

A PF-map $f: P \rightarrow F$ will be called a $P \mathcal{F}_{p}$-map if $F \in \mathcal{F}_{p}$.

THEOREM 6.1. For each prime $p$, every $P \in \mathcal{P}$ has a special right h-universal $\mathcal{P F}$-map $u_{p}: P \rightarrow F_{p}(P)$ having the following additional properties.

(a) $u_{p}$ is an inclusion map.

(b) $F_{p}(P)$ has a chain homotopy $S$ over $K G_{p}$ which is 0 on $P$ and which contracts $F_{p}(P)$ to $P$. 
(c) ("The strong homotopy extension property"). Let $g_{1}, g_{2}: F_{p}(P) \rightarrow F$ be maps in $\mathcal{F}_{p}$. Let $S$ be a PF-homotopy between $g_{1} u_{p}$ and $g_{2} u_{p}$. Then there is an F-homotopy $D$ between $g_{1}$ and $g_{2}$ such that $D u_{p}=S$.

This theorem will be proved in $\S 10$.

An immediate consequence of this theorem is that we can factor the map $\nabla: \otimes^{n} C(X) \rightarrow C\left(X^{n}\right)$ as $\nabla=g_{p} u_{p}$ where $g_{p}: F_{p}\left(\otimes^{n} C(X)\right) \rightarrow C\left(X^{n}\right)$ is unique up to F-homotopy.

This map $g_{p}$ is clearly a natural transformation of prefunctors because a semisimplicial map $f: X \rightarrow Y$ induces a commutative diagram

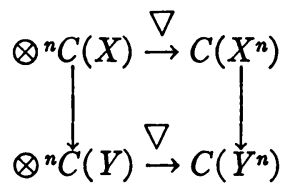

The corresponding diagram with $F_{p}$ applied to $\otimes^{n} C(X)$ and $\otimes^{n} C(Y)$ then commutes up to homotopy by the $h$-universal property of $u_{p}$.

Theorem 6.2. $\left(g_{p} / \pi_{p}\right)_{*}: H\left(F_{p}\left(\otimes^{n} C(X)\right) / \pi_{p}\right) \rightarrow H\left(C\left(X^{n}\right) / \pi_{p}\right)$ is an isomorphism.

If $n$ is a power of $p$, this theorem gives us the required homology $H\left(C P^{n} X\right)$. The general case will be treated in $\S 12$ by reducing it to Theorem 6.2. The theorem itself will be proved in $\$ 18$.

The methods used in proving this theorem also give another result which will be used in $\$ 13$. In order to state this result, it is necessary to define a subcategory $P_{0}$ of $P$.

Definition 6.5. $P_{0}$ shall be the full subcategory of $P$ with objects of the following forms.

$$
\sum_{i \bmod r} \otimes^{n / r}\left(M_{i} \otimes M_{i+1} \otimes \cdots \otimes M_{i+r-1}\right)
$$

where $M_{0}, \cdots, M_{r-1}$ are disjoint $K$-free chain complexes. Here $r$ is any divisor of $n$, and the subscripts on the $M_{i}$ are to be reduced modulo $r$. The group $\pi$ acts, as usual by cyclic permutation.

(b) All finite direct sums of objects listed under (a).

Lemma 6.1. If $P \in \mathcal{P}_{0}$ and $H(P)=0$, then $H\left(F_{p}(P) / \pi_{p}\right)=0$.

This will be proved in $\$ 16$.

REMARK 6.1. If $\pi^{\prime}$ is any subgroup of $\pi$ and $P \in \mathcal{P}_{0}$, then $P$, considered as a $K \pi^{\prime}$-complex, is in the category $\mathcal{P}_{0}$ defined for the group $\pi^{\prime}$.

This remark forms the basis for an inductive proof of Lemma 6.1.

7. Weak injectivity. Let $\phi: \Lambda \rightarrow \Gamma$ be a map of rings. Then every $\Gamma$-module can be made into a $\Lambda$-module by means of $\phi[2$, Chapter II, §6]. 
Definition 7.1. Let $Q$ and $A$ be left $\Gamma$-modules. We say $Q$ is $\phi$-injective relative to $A$ if the following condition holds for every $\Gamma$-submodule $B \subset A$.

(*) Let $f: B \rightarrow Q$ be a $\Gamma$-map. If $f$ extends to $A$ as a $\Lambda$-map then it also extends to $A$ as a $\Gamma$-map.

We say $Q$ is $\phi$-injective if this condition holds for all $A$.

The notion of $\phi$-projective is defined dually.

Definition 7.2. Let $Q$ and $A$ be left $\Gamma$-modules. We say $Q$ is weakly $\phi$-injective relative to $A$ if the following condition holds.

$\left.{ }^{* *}\right)$ Let $B \subset A$ be a sub- $\Gamma$-module which is a direct summand when $A$ is regarded as a $\Lambda$-module. Then every $\Gamma$-map $B \rightarrow Q$ extends to a $\Gamma$-map $A \rightarrow Q$.

We say $Q$ is weakly $\phi$-injective if this condition holds for all $A$.

Weakly $\phi$-projective is defined dually.

REMARK 7.1. Weakly $\phi$-injective is the same as the notion of $(\Gamma, \phi(\Lambda))$ injective of [12]. The absolute notions $\phi$-injective and weakly $\phi$-injective are easily seen to be equivalent and to be equivalent to the notion $\phi$-injective of [2, Chapter II, §6]. In fact, $Q$ is imbedded naturally as a $\Lambda$-direct summand of $\operatorname{Hom}_{\Lambda}(\Gamma, Q)$. Therefore, if $Q$ is weakly $\phi$-injective, it is $\phi$-injective in the Cartan-Eilenberg sense. Suppose now that $Q$ is $\phi$-injective in the CartanEilenberg sense. If $g: A \rightarrow Q$ is a $\Lambda$-map extending $f: B \rightarrow Q$, we blow up $g$ to a $\Gamma$-map $g^{\prime}: A \rightarrow \operatorname{Hom}_{\Lambda}(\Gamma, Q)$ by $g^{\prime}(a) \cdot(\gamma)=g(\gamma a)$. Composing $g^{\prime}$ with the $\Gamma$ retraction $\operatorname{Hom}_{\Lambda}(\Gamma, Q) \rightarrow Q$ gives a $\Gamma$-extension of $f$. This shows that $Q$ is $\phi$-injective in our sense.

We now generalize the theorem on mapping an acyclic right complex into an injective right complex [2, Chapter V, Proposition 1.1a].

Proposition 7.1. Let $A$ be $a \Gamma$-module and $X$ a right $\Gamma$-complex over $A$ such that $X$ has a contracting homotopy which is a $\Lambda$-map. Let $Y$ be a right $\Gamma$-complex over a $\Gamma$-module $B$. Then,

(a) If each $Y^{i}$ is weakly $\phi$-injective relative to $X^{i}$, any $\Gamma$-map $f: A \rightarrow B$ extends to a $\mathrm{\Gamma}$-map $\mathrm{g}: X \rightarrow Y$.

(b) If each $Y^{i}$ is weakly $\phi$-injective relative to $X^{i+1}$, then any $\Gamma$-map $g: X \rightarrow Y$ such that $g \mid A=0$ is $\Gamma$-homotopic to 0 by a homotopy $S$ with $S \mid X^{0}=0$.

Proof. In (a), suppose $g \mid X^{i}$ has been defined for $i<n$. Then $g$ is determined on the image of $\delta: X^{n-1} \rightarrow X^{n}$. The existence of a $\Lambda$-homotopy $D$ contracting $X$ implies that this image is a $\Lambda$-direct summand of $X^{n}$. In fact, $\delta D$ is a retraction. Therefore, we can extend $g$ to all of $X^{n}$ using the weak $\phi$ injectivity of $Y^{n}$ with respect to $X^{n}$.

The proof of (b) is similar.

There is an obvious dual involving left complexes and weak $\phi$-projectivity.

I will now give some sufficient conditions for relative weak injectiveness for a particular map $\phi$. As usual, $\pi$ will be a cyclic group of order $n, \pi_{p}$ the Sylow $p$-group of $\pi$, and $G_{p}$ the unique complement of $\pi_{p}$ in $\pi$. The map in question is $\phi_{p}: K G_{p} \rightarrow K \pi$ induced by the inclusion $G_{p} \subset \pi$. I will write " $p$-injective" in place of " $\phi_{p}$-injective." 
Lemma 7.1. Let $Q$ be a $K \pi$-module which is $K$-free and has a base permuted up to sign by $\pi$. Assume $\pi_{p}$ acts freely on this base. Then $Q$ is p-injective.

Proof. If $A$ and $B$ are $K \pi$-modules and $f: A \rightarrow B$ is a $K$-map (not necessarily $\pi$-equivariant), the $\pi_{p}$-norm of $f$ is defined to be $N_{p}(f)=\sum t f t^{-1}$, the sum being over all $t \in \pi_{p}$. If $f$ is a $K G_{p}$-map, then $N_{p}(f)$ is clearly a $K \pi$-map.

I will now show that there is a $K G_{p}$-endomorphism $\theta$ of $Q$ such that $N_{p}(\theta)=1$ (the identity map) (cf. [2, Chapter XII, Proposition 1.1]). The given base for $Q$ falls into orbits under the action of $\pi$ (ignoring signs for the moment). Choose one element $e_{i}$ in each orbit. Then $Q$ is generated over $K$ by the elements $x t e_{i}$ with $x \in G_{p}$ and $t \in \pi_{p}$. Define $\theta: Q \rightarrow Q$ by $\theta\left(x e_{i}\right)=x e_{i}$ for $x \in G_{p}$, and $\theta\left(x t e_{i}\right)=0$ if $t \neq 1, x \in G_{p}, t \in \pi_{p}$. Then $\theta$ is a well defined $K G_{p^{-}}$ map because of the freeness condition and because the orders of $G_{p}$ and $\pi_{p}$ are relatively prime. Clearly $N_{p}(\theta)=1$.

Now, let $B$ be a $K \pi$-submodule of a $K \pi$-module $A$. Let $f: B \rightarrow Q$ be a $K \pi$-map which is extendible to a $K G_{p}$-map $g: A \rightarrow Q$. Define $h: A \rightarrow Q$ by $h=N_{p}(\theta g)$. Then $h$ is a $K \pi$-map and $h \mid A=N_{p}(\theta f)=N_{p}(\theta) f=f$.

Lemma 7.2. Let $\pi^{\prime}$ be any subgroup of $\pi_{p}$. Let $Q$ be a $K \pi$-module having a $K$-base $\left\{e_{i}\right\}$ permuted up to sign by $\pi$. Assume that if $t \in \pi_{p}$ and $t e_{i}= \pm e_{i}$ for some $i$, then $t \in \pi^{\prime}$ and $t e_{i}=e_{i}$.

Let $A$ be a Kr-module with all its elements fixed under $\pi^{\prime}$. Then $Q$ is weakly p-injective relative to $A$.

Proof. Let $Q_{0} \subset Q$ be the submodule of elements fixed under $\pi^{\prime}$. If $B \subset A$ and $f: B \rightarrow Q$ is a $K \pi$-map, then $\operatorname{im} f \subset Q_{0}$. Consequently, it is sufficient to show that $Q_{0}$ is $p$-injective as a $K\left(\pi / \pi^{\prime}\right)$-module, because, if $B$ is a $K G_{p}$-direct summand, we can then extend $f$ to a $K\left(\pi / \pi^{\prime}\right)$-map $A \rightarrow Q_{0}$ which will automatically be a $K \pi$-map. I claim that $Q_{0}$ satisfies the conditions of Lemma 7.1. It is sufficient to verify this in case $Q$ is generated over $K \pi$ by a single element $e$. Let $\pi^{\prime \prime}$ be the set of all $t$ in $\pi_{p}$ such that $t e=e$. Then $\pi^{\prime \prime} \subset \pi^{\prime}$. But $Q$, considered as a $K\left(\pi / \pi^{\prime \prime}\right)$-module, is now free with respect to $\pi_{p} / \pi^{\prime \prime}$. The assertion is obvious in this case.

8. Partial $p$-resolutions. I will now define a special type of right complex. Let $\pi_{p}$ be the Sylow $p$-group of $\pi$ and let

$$
0=\pi_{p}^{(0)} \subset \pi_{p}^{(1)} \subset \cdots \subset \pi_{p}^{(h)}=\pi_{p}
$$

be its composition series. Let $A$ be any $K \pi$-module and $r$ a non-negative integer. Regard $A$ as a graded module whose components are zero except in dimension $r$.

Definition 8.1. A partial $p$-resolution of $(A, r)$ is a right complex $W$ over $A$, indexed as follows

$$
0 \rightarrow A \rightarrow W_{r} \rightarrow W_{r-1} \rightarrow \cdots
$$


satisfying the following conditions.

(a) $W$ has a contracting homotopy which is a $K G_{p}$-map.

(b) Each $W_{i}$ has a base $\left\{e_{j}^{i}\right\}$ permuted up to sign by $\pi$.

(c) If $t \in \pi_{p}$, we cannot have $t e_{j}^{i}=-e_{j}^{i}$.

(d) Let $t \in \pi_{p}^{(\mathbf{k})}$. Then $t e_{j}^{i}=e_{j}^{i}$ if and only if $i \leqq r / p^{k}$.

REMARK 8.1. We may reformulate conditions (b), (c), and (d) as follows: $W \in \mathcal{F}_{p}$ and $\pi_{p}^{(k)}$ acts trivially on $W_{i}$ if $i \leqq r / p^{k}$.

The filtration used on $W$ in the statement " $W \in \mathcal{F}_{p}$ " is, of course, given by letting $\Phi_{k} W$ be the complex $W_{k} \rightarrow W_{k-1} \rightarrow \cdots$ for $k \leqq r$. For $k>r$, we let $\Phi_{k} W=W$.

REMARK 8.2. Let $\pi^{\prime}$ be any subgroup of $\pi$. Then a partial $p$-resolution $W$ of a $K \pi$-module $A$ is also a partial $p$-resolution for $A$ if $A$ is considered as a $K \pi^{\prime}$-module. This remark is the basis for the inductive proof of Theorem 6.2 and Lemma 6.1.

REMARK 8.3. If $\pi$ is cyclic of prime order $p$ and $\Delta^{m}$ is the standard $m$ simplex, the complex $C\left(\left(\Delta^{m}, \dot{\Delta}^{m}\right)^{p}\right)$ is a partial $p$-resolution over $(Z, p m)$. This was the original motivation for the construction to be given in the next two sections.

Lemma 8.1. Let $P \in \mathcal{P}$. Then, for each $r,\left(P_{r}, r\right)$ has a partial p-resolution $W$ such that $W_{i}=0$ for $i<r / p^{h}$ where $p^{h}$ is the order of $\pi_{p}$.

Proof. By definition, $P_{r}$ has a base $\left\{e_{i}\right\}$ permuted up to sign by $\pi$, such that if $t e_{i}= \pm e_{i}$ and $t$ generates $\pi_{p}^{(k)}$, then $p^{k} \mid r$ and $t e_{i}=e_{i}$ unless $p=2, t \neq 1$, and $r / 2^{k}$ is odd in which case $t e_{i}=-e_{i}$. It is clearly sufficient to consider the case where $P_{r}$ is generated over $K \pi$ by a single element $e$.

It is sufficient to prove the lemma for the case $\pi=\pi_{p}$. For, suppose the lemma has been proved in this case. We then let $P_{r}^{\prime}$ be the sub- $K \pi_{p}$-module of $P_{r}$ generated by $e$. Let $G_{p}^{\prime}$ be the set of elements $g$ of $G_{p}$ such that $g e= \pm e$, and let $1=g_{1}, \cdots, g_{m}$ be coset representatives for $G_{p} / G_{p}^{\prime}$. Then $P_{r}$ is the direct sum of the $K \pi_{p}$-submodules $g_{i} P_{r}^{\prime}$. We now take a partial $p$-resolution $W^{\prime}$ for $P_{r}^{\prime}$ as a $K \pi_{p}$-module. Take an isomorphic copy of $W^{\prime}$ as a partial $p$-resolution for $g_{i} P_{r}^{\prime}$. Call this resolution $g_{i} W^{\prime}$. The required $W$ is now the direct sum of the $g_{i} W^{\prime}$. The elements $g_{i}$ of $G_{p}$ act on $W$ in the obvious way by permuting the $g_{i} W^{\prime}$. If $g \in G_{p}^{\prime}$, we make $g$ act trivially on $W$ if $g e=e$ and make it act by changing all signs if $g e=-e$. A contracting homotopy for $W^{\prime}$ gives a $K G_{p^{-}}$ homotopy for $W$.

We are now reduced to the case where $P_{r}$ has a single generator $e$ and $\pi=\pi_{p}$. In this case, $W$ will be given by explicit formulas. Let $k$ be the largest integer such that $t \in \pi_{p}^{(k)}$ implies $t e= \pm e$. Let $W_{s}=0$ if $s<r / p^{k}$. If $s \geqq r / p^{k}$, $W_{s}$ will have one generator $\alpha_{s}$ with the relations that $\alpha_{s}$ is fixed under $\pi_{p}^{(i)}$ if and only if $s \leqq r / p^{i}$. Let $T$ generate $\pi$. Then $T p^{h-i}$ generates $\pi_{p}^{(i)}$. Thus, the relations on $\alpha_{s}$ reduce to the single relation $T^{p-i} \alpha_{s}=\alpha_{s}$ where $i$ is the largest integer such that $s \leqq r / p^{i}$. 
We must now define the maps $\epsilon: A \rightarrow W_{r}$ and $\partial: W_{s} \rightarrow W_{s-1}$. There are two cases to consider.

CASE 1. $p$ odd or $r / p^{k}$ even. In this case we define $\epsilon: A \rightarrow W_{r}$ by

$$
\epsilon(e)=\left(1+T^{p^{h-k}}+T^{2 p^{h-k}}+\cdots+T^{\left(p^{k-1}\right) p^{h-k}}\right) \alpha_{r}
$$

and define $\partial: W_{s} \rightarrow W_{s-1}$ by

$$
\begin{aligned}
& \partial \alpha_{s}=\left(T^{p^{h-k}}-1\right) \alpha_{s-1} \text { if } s-r \text { is even, } \\
& \partial \alpha_{s}=\left(1+T^{p^{h-k}}+T^{2 p^{h-k}}+\cdots+T^{\left(p^{i-1)} p^{h-k}\right.}\right) \alpha_{s-}
\end{aligned}
$$

if $s-r$ is odd. Here $i$ is the smallest integer such that $T^{p^{h-k+i}} \alpha_{s-1}=\alpha_{s-1}$.

CASE 2. $p=2$ and $r / 2^{k}$ odd. In this case, we merely substitute $-T^{n-k}$ for $T^{p^{h-k}}$ in the above formulas.

We leave to the reader the trivial verification that these complexes are acyclic. The existence of a contracting homotopy follows from this and the fact that im $\epsilon$ and im $\partial$ are $K$-direct summands (because $\epsilon(e)$ and $\partial \alpha_{s}$ are combinations of basis elements with integral coefficients whose g.c.d. is 1).

REMARK 8.4. The main difficulty in extending the theory to noncyclic groups $\pi$ is that of constructing a complex analogous to the above $W$.

REMARK 8.5. Note that if $P$ has no basis element fixed up to sign under $\pi_{p}^{(i)}$ then neither has $W$.

9. Attaching one complex to another. In this section, I will show how to replace one of the modules occurring in a complex by another complex. This is the basic construction used in the proof of Theorem 6.1.

Assume we have again a map of rings $\phi: \Lambda \rightarrow \Gamma$. Let $A$ be a $\Gamma$-complex

$$
\cdots \rightarrow A_{N+1} \rightarrow A_{N} \rightarrow A_{N-1} \rightarrow \cdots \text {. }
$$

Suppose we are given a right $\Gamma$-complex

$$
0 \rightarrow A_{N} \rightarrow W_{N} \rightarrow W_{N-1} \rightarrow \cdots
$$

over $A_{N}$ which has a contracting homotopy over $\Lambda$, i.e. a chain homotopy $D$ over $\Lambda$ such that $\partial D+D \partial=1$.

Finally, suppose we have a map $\psi: W \rightarrow A$ of degree -1 making the diagram

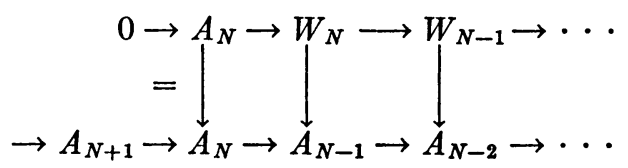

commute. Such a map will be called an "attaching map." It will exist if $A$ satisfies the following condition.

Condition 9.1. For $n<N, A_{n}$ is weakly $\phi$-injective relative to $W_{n+1}$.

If this is satisfied, the existence of $\psi$ follows from Proposition 7.1.

We now define a new complex $A^{*}$ by replacing $A_{N}$ by $W$. Let $A_{n}^{*}=A_{n}$ if 
$n>N$. Let $A_{N}^{*}=W_{N}$. Let $A_{n}^{*}=A_{n}+W_{n}$ if $n<N$. The boundary operator $\partial^{*}$ in $A^{*}$ is defined as follows.

In dimensions $>N+1, \partial^{*} a=\partial a$,

In dimension $N+1, \partial^{*} a=\epsilon \partial a$,

In dimension $N, \partial^{*} w=(\psi(w), \partial w)$,

In dimensions $n<N, \partial^{*}(a, w)=\left(\partial a+(-1)^{N-n} \psi(w), \partial w\right)$.

It is trivial to verify that $\partial^{*} \partial^{*}=0$. There is a natural inclusion $A \subset A^{*}$ of $A$ as a subcomplex of $A^{*}$.

This construction has three basic properties given in the next three lemmas.

Lemma 9.1. $A^{*}$ has a $\Lambda$-homotopy $S$, zero on $A$, which contracts $A^{*}$ to $A$.

By a homotopy contracting $A^{*}$ to $A$, I mean a chain homotopy $S: A^{*} \rightarrow A^{*}$ such that $1-(\partial S+S \partial)$ is a retraction of $A^{*}$ on $A$, i.e. its image is $A$ and it is the identity map on elements of $A$.

Proof. Let $D$ be the contracting homotopy for the complex

$$
0 \rightarrow A_{N} \rightarrow W_{N} \rightarrow W_{N-1} \rightarrow \cdots \text {. }
$$

Define $S\left|W_{i}=D\right| W_{i}$ for $i \neq N, S \mid W_{N}=0$, and $S \mid A=0$. This obviously has the required properties.

Lemma 9.2. Let $B$ be a $\Gamma$-complex. Assume that for $n \leqq N, B_{n}$ is weakly $\phi$-injective relative to $W_{n}$. Then any $\Gamma$-map $f: A \rightarrow B$ extends to a $\Gamma$-map $f^{*}: A^{*} \rightarrow B$.

Proof. Let $f^{*}=f$ in dimensions $>N$. In dimension $N$, let $f_{N}^{*}$ be any $\Gamma$-map $W_{N} \rightarrow B_{N}$ extending $f_{N}: A_{N} \rightarrow B_{N}$. This exists since $A_{N}$ is a $\Lambda$-direct summand of $W_{N}$ (because of the contracting homotopy). Now, suppose $k<N$ and $f_{s}^{*}$ has been defined for $s>k$ so that $\partial f_{s+1}=f_{s} \partial^{*}$. Consider the diagram

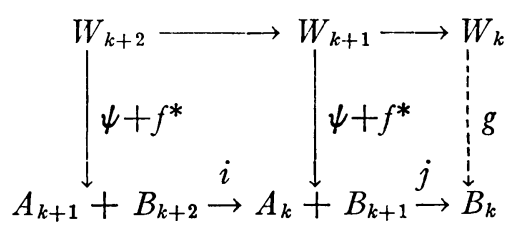

Here, $i$ and $j$ are defined by

$$
\begin{aligned}
& i(a, b)=\left(\partial a,(-1)^{N-k+1} f(a)+\partial b\right), \\
& j(a, b)=(-1)^{N-k} f(a)+\partial b .
\end{aligned}
$$

The map $\psi+f^{*}$ sends $w$ into $\left(\psi(w), f^{*}(w)\right)$. In case $k=N-1$, the term $W_{k+2}$ should be replaced by $A_{N}$ and this should be mapped into $A_{N}+B_{N+1}$ by sending $a$ to $(a, 0)$.

It is easy to verify that the diagram commutes and $j i=0$. Consequently, 
we can fill in the dotted line with a $\Gamma$-map $g$. Now, define $f^{*}$ in dimension $k$ by $f^{*}(a, w)=f(a)+g(w)$.

Lemma 9.3. Let $B$ be a $\Gamma$-complex. Assume that for each $n \leqq N, B_{n+1}$ is weakly $\phi$-injective relative to $W_{n}$. Suppose $f^{*}, g^{*}: A^{*} \rightarrow B$ are given extensions of maps $f, g: A \rightarrow B$. Let $S$ be a $\Gamma$-homotopy between $f$ and $g$. Then $S$ extends to a $\Gamma$-homotopy $S^{*}$ between $f^{*}$ and $g^{*}$.

Proof. Let $h=f-g, h^{*}=f^{*}-g^{*}$ so $S: h \simeq 0$. Define a $\Gamma$-homotopy $S_{0}^{*}: A^{*} \rightarrow B$ by $S_{0}^{*}\left|A=S, S_{0}^{*}\right| W_{k}=0$ for $k \neq n$, and $S_{0}^{*} \mid W_{N}=$ any $\Gamma$-map extending $S \mid A_{N}$. Then $S_{0}^{*}: h^{*} \simeq h_{0}^{*}$ where $h_{0}^{*} \mid A=0$. This implies that $h_{0}^{*} \mid W$ is a chain map $W \rightarrow B$ such that $h_{0}^{*} \mid A_{N}=0$. By Proposition 7.1 , there is a homotopy $S_{1}: h_{0}^{*} \mid W \simeq 0$ with $S_{1} \mid A_{N}=0$. Define $S_{1}^{*}: A^{*} \rightarrow B$ by $S_{1}^{*} \mid W=S_{1}$ and $S_{1}^{*} \mid A=0$. Then $S_{1}^{*}: h_{0}^{*} \simeq 0$. Now set $S^{*}=S_{0}^{*}+S_{1}^{*}$. This gives the required homotopy.

10. Proof of Theorem 6.1. Let $P \in P$. We will construct $F_{p}(P)$ as the union of an increasing sequence of complexes $F_{p}^{(k)}(P)$ starting with $F_{p}^{(-1)}(P)=P$. The universal map $u$ will be the inclusion $P=F_{p}^{(-1)}(P) \subset F_{p}(P)$. Each $F_{p}^{(k)}(P)$ will be filtered and, for $k \geqq r, \Phi_{r} F_{p}^{(k)}(P)$ will be independent of $k^{\circ}$ and so will be $\Phi_{r} F_{p}(P)$. In addition, for $r \geqq k, \Phi_{r} F_{p}^{(k)}(P)$ will consist of all elements of dimension $\leqq r$. We make the inductive assumption that $F_{p}^{(k-1)}(P)$ has been defined and that for $r \leqq k-1, \Phi_{r} F_{p}^{(k-1)}(P)$ has the properties given in the definition of the category $\mathcal{F}_{p}$.

Let $W^{(k)}$ be a partial $p$-resolution of $\left(P_{k}, k\right)$. This exists by Lemma 8.1. Since $\Phi_{k-1} F_{p}^{(k-1)}(P)$ consists of all elements of dimension $\leqq k-1$ and satisfies the conditions imposed on $\Phi_{k-1}$ in the definition of the category $F_{p}$, we verify immediately, using Lemma 7.2, that Condition 9.1 is satisfied. The map $\phi$ used here is, of course, $\phi_{p}: K G_{p} \rightarrow K \pi$. We now define $F_{p}^{(k)}(P)$ to be the result of attaching $W^{(k)}$ to $F_{p}^{(k-1)}(P)$ by the process of $\S 9$. The filtration on $F_{p}^{(k)}(P)$ is determined automatically by the requirements stated in the first paragraph of the present section. Namely, $\Phi_{r} F_{p}^{(k)}=\Phi_{r} F_{p}^{(k-1)}$ for $r<k$ while $\Phi_{r} F_{p}^{(k)}$ consists of all elements of dimension $\leqq r$ if $r \geqq k$. It follows from the definition of a partial $p$-resolution that $\Phi_{k} F_{p}^{(k)}(P)$ now satisfies the conditions imposed on $\Phi_{k}$ in the definition of the category $\mathcal{F}_{p}$ because $\Phi_{k} F_{p}^{(k)}$ is the direct sum of $\Phi_{k-1} F_{p}^{(k)}$ and $W^{(k)}$. Therefore, we may iterate the process to get $F_{p}^{(k)}(P)$ for all $k$. We then define $F_{p}(P)$ to be the union of all $F_{p}^{(k)}(P)$.

The fact that $u_{p}: P \rightarrow F_{p}(P)$ is a special $h$-universal map and satisfies the strong homotopy extension condition follows immediately from the results of $\S 9$. For, suppose $f: P \rightarrow A$ is a PF-map and that $f^{(k)}: F_{p}^{(k)}(P) \rightarrow A$ has been defined so as to preserve filtration. Then $f^{(k)}: \Phi_{k+1} F_{p}^{(k)}(P) \rightarrow \Phi_{k+1} A$. The conditions of Lemma 9.2 are clearly satisfied because of Lemma 7.2. Therefore, we can extend $f^{(k)}$ to $f^{(k+1)}: F_{p}^{(k+1)}(P) \rightarrow A$ by extending $f^{(k)} \mid \Phi_{k+1} F_{p}^{(k)}(P)$ as a map into $\Phi_{k+1} A$. If $f, g: F_{p}(P) \rightarrow A$ are such that there is a homotopy $S: f u_{p} \simeq g u_{p}$, we extend $S$ inductively to a homotopy $S^{\prime}: f \simeq g$ in exactly the way in which 
we extended $f$. Here, of course, we use Lemma 9.3 in place of Lemma 9.2. This proves the strong homotopy extension property. The uniqueness condition in the definition of $h$-universal maps is an immediate consequence of this property.

We must now show that $F_{p}(P)$ has a $K G_{p}$-homotopy $S$, zero on $P$, such that $S$ contracts $F(P)$ to $P$. Assume we have defined such a homotopy $S^{(k)}$ on $F_{p}^{(k)}(P)$. Let $D$ be a contracting homotopy for $W^{(k+1)}$ over $K G_{p}$. Then $D \mid W_{k+1}=0$ and so $D \mid A_{k+1}=0$. Therefore, we can define $S^{(k+1)}$ on $F_{p}^{(k+1)}(P)$ by $S^{(k+1)} \mid F_{p}^{(k)}(P)=S^{(k)}$ and $S^{(k+1)} \mid W=D$. Then $S^{(k+1)}$ is zero on $P$ and contracts $F_{p}^{(k+1)}(P)$ to $P$. We let $S$ be the direct limit of the $S^{(k)}$.

This concludes the proof of the theorem. We conclude this section by observing some further properties of $F_{p}(P)$ which follow immediately from the construction.

REMARK 10.1. Suppose $\pi^{\prime}$ is a subgroup of $\pi$. Then the above $u_{p}: P \rightarrow F_{p}(P)$ satisfies all the conditions of Theorem 6.1 for the group $\pi^{\prime}$ as well as for the group $\pi$. This follows immediately from Remark 8.2 and the fact that the construction of $F_{p}(P)$ over $\pi$ and over $\pi^{\prime}$ is exactly the same except that we must choose our attaching maps more carefully over $\pi$ than over $\pi^{\prime}$ in order to make them $\pi$ equivariant.

REMARK 10.2. Suppose the partial $p$-resolutions $W$ are chosen as in Lemma 8.1. Then the $s$-dimensional part of $F_{p}^{(\mathbf{k})}(P)$ will be constant for $k>p^{h} s$ where $p^{h}$ is the order of $\pi_{p}$. Consequently, if $P$ and $P^{\prime}$ are isomorphic in dimensions $\leqq r$, we may choose $F_{p}(P)$ and $F_{p}\left(P^{\prime}\right)$ to be isomorphic in dimensions $\leqq r / p^{h}$. Similarly, if two maps $P \rightarrow F$ and $P^{\prime} \rightarrow F$ agree in dimensions $\leqq r$, we may choose the factoring maps $F_{p}(P) \rightarrow F$ and $F_{p}\left(P^{\prime}\right) \rightarrow F$ to agree in dimensions $\leqq r / P^{h}$ (we are still assuming, of course, that $P$ and $P^{\prime}$ are isomorphic in dimensions $\leqq r)$.

REMARK 10.3. When regarded as a filtered module, $F_{p}(P)$ is clearly isomorphic to the direct sum $\sum W^{(k)}$. The inductive construction is only needed to define the boundary operator. The filtration is given by $\Phi_{k} \sum W^{(k)}$ $=\sum_{r \leq k} W^{(r)}$. The map $u_{p} \mid P_{r}$ is just the augmentation $\epsilon: P_{r} \rightarrow W_{r}^{(r)}$. Consequently, to determine the module extension $P \subset F_{p}(P)$, it is sufficient to determine the extensions $P_{r} \subset W_{r}^{(r)}$.

REMARK 10.4. If any $P_{r}=0$, we may obviously choose $W^{(r)}=0$. Therefore, if $P$ is zero in dimensions greater than some $N$, we may choose $F_{p}(P)$ to have the same property.

11. Transfer theorems. In this section, the groups involved need not be cyclic.

Suppose $\pi$ is a subgroup of a group $\Pi$. Let $A$ be a module on which $\Pi$ operates. There is an obvious quotient map $\eta: A / \pi \rightarrow A / \Pi$. We now define the transfer (cf. [2, Chapter XII, §8]) $\phi: A / \Pi \rightarrow A / \pi$ as follows.

The group $\Pi$ is the union of disjoint right cosets $\Pi=U_{\pi g_{i}}$. If $x \in A / \Pi$, choose a representative $y \in A$ of $x$ and let $\phi(x)$ be the element of $A / \pi$ represented by $\sum g_{i} y$. 
It is easy to check that $\phi$ is independent of the choice of the representative $x$ and of the choice of the coset representatives $g_{i}$.

Clearly, $\eta \phi(x)=[\Pi: \pi] x$ for all $x \in A / \Pi$. If $\pi$ happens to be normal in $\Pi$, then $\Pi / \pi$ acts on $A / \pi$ and it is easy to see that $\phi \eta(x)=\sum t x$ for all $x \in A / \pi$, the sum being taken over all $t \in \Pi / \pi$.

The maps $\eta$ and $\phi$ are obviously natural.

Now, let $R$ be any set of primes. Let $\mathcal{C}$ be the Serre class [20] of all torsion groups whose $p$-primary components are zero when $p \in R$. Let $\pi$ be a finite group and let $\pi_{p}$ be a Sylow $p$-group of $\pi$ for each prime $p$. Let $n$ be the order of $\pi$.

Theorem 11.1. Let $A$ and $B$ be chain complexes on which $\pi$ acts. Let $f: A \rightarrow B$ be a $\pi$-equivariant chain map. Assume that for each $p \in R$ such that $p \mid n$, the map

$$
\left(f / \pi_{p}\right)_{*}: H\left(A / \pi_{p}\right) \rightarrow H\left(B / \pi_{p}\right)
$$

is a e-isomorphism [20]. Then,

$$
(f / \pi)_{*}: H(A / \pi) \rightarrow H(B / \pi)
$$

is also a e-isomorphism.

Proof. Let $\eta_{p}$ and $\phi_{p}$ be the quotient and transfer maps corresponding to the subgroup $\pi_{p}$ of $\pi$. These maps are chain maps since $\eta$ and $\phi$ are natural.

Let $n=\prod p^{r_{p}}$ be the prime factorization of $n$. Let $d$ be the greatest common divisor of the numbers $n / p^{r p}$ for $p \in R$. Then $d$ is a product of primes not in $R$. Choose integers $a_{p}$ for $p \in R$ and $p \mid n$ such that $d=\sum a_{p} n / p^{r_{p}}$.

Let $A^{\prime}=\sum A / \pi_{p}$, the sum being taken over those $p$ such that $p \mid n$ and $p \in R$. Define $\eta^{\prime}: A^{\prime} \rightarrow A / \pi$ to be the map having the value $\eta_{p}$ on $A / \pi_{p}$. Define $\phi^{\prime}: A / \pi \rightarrow A^{\prime}$ to be the map whose component on $A / \pi_{p}$ is $a_{p} \phi_{p}$. It is easy to see that $\eta^{\prime} \phi^{\prime}(x)=d x$ for all $x \in A / \pi$.

In the same way we define $B^{\prime}$ and its $\eta^{\prime}$ and $\phi^{\prime}$ using the same integers $a_{p}$ in the definition of $\phi^{\prime}$ which we used for $A^{\prime}$.

Let $f^{\prime}: A^{\prime} \rightarrow B^{\prime}$ be the map induced by $f$. Then $(f / \pi) \eta^{\prime}=\eta^{\prime} f^{\prime}$ and $\phi^{\prime}(f / \pi)$ $=f^{\prime} \phi^{\prime}$. Also, the kernel and cokernel of $f_{*}^{\prime}: H\left(A^{\prime}\right) \rightarrow H\left(B^{\prime}\right)$ are both in $\mathrm{C}$. Let $R^{\prime}=\operatorname{ker} f_{*}^{\prime}$ and $Q^{\prime}=\operatorname{ckr} f_{*}^{\prime}$. Let $R=\operatorname{ker}(f / \pi)_{*}$ and $Q=\operatorname{ckr}(f / \pi)_{*}$. Then we have

$$
\begin{aligned}
& R \stackrel{\phi_{*}^{\prime}}{\longrightarrow} R^{\prime} \stackrel{\eta_{*}^{\prime}}{\longrightarrow} R \\
& Q \stackrel{\phi_{*}^{\prime}}{\longrightarrow} Q^{\prime} \stackrel{\eta_{*}^{\prime}}{\longrightarrow} Q .
\end{aligned}
$$

In each case, the composition is multiplication by $d$. Since $R^{\prime}, Q^{\prime} \in \mathcal{C}$, it follows that $R, Q \in \mathcal{C}$. To see this, let $x \in R$. Then $\phi_{*}^{\prime}(x)$ has finite order $k$ where no prime in $R$ divides $k$. Therefore, $d k x=\eta_{*}^{\prime} k \phi_{*}^{\prime}(x)=0$. But, no prime in $R$ divides $d k$. 
REMARK 11.1. If we know that every element of $\operatorname{ker} f_{*}^{\prime}$ has order dividing some fixed integer $k$, it follows that every element of $\operatorname{ker}(f / \pi)_{*}$ has order dividing $d k$. The same applies to the cokernels.

REMARK 11.2. The above proof also yields the following result. If $\left(f / \pi_{p}\right)_{*}$ is zero $\bmod \mathfrak{C}$ for all $p \in \mathbb{R}$ such that $p \mid n$, then $(f / \pi)_{*}$ is zero $\bmod \mathfrak{C}$. To see this, let $I$ be the image of $(f / \pi)_{*}$ and let $I^{\prime}$ be the image of $f_{*}^{\prime}$. Then $I^{\prime} \in \mathrm{e}$ and the above argument applied to $I$ and $I^{\prime}$ instead of to $R$ and $R^{\prime}$ shows that $I \in \mathbb{e}$.

The next theorem and its proof are due essentially to Richardson [17]. Suppose $\pi$ is a finite group which acts on a chain complex $A$. The quotient map $\eta: A \rightarrow A / \pi$ induces a map $\eta_{*}: H(A) \rightarrow H(A / \pi)$. If we let $\pi$ act trivially on $A / \pi$, then $\eta$ is $\pi$-equivariant and consequently so is $\eta_{*}$. It follows that $\eta_{*}$ induces a unique map $\eta_{*}^{\prime}: H(A) / \pi \rightarrow H(A / \pi)$.

TheOREM 11.2. Let $n$ be the order of $\pi$. Then $n$ annihilates the kernel and cokernel of $\eta^{\prime}$.

Proof. Let $\phi: A / \pi \rightarrow A$ be the transfer map corresponding to the trivial subgroup of $\pi$. Then $\eta \phi(x)=n x$. Also $\phi \eta(y)=\sum t y$, the sum being over all $t$ in $\pi$. Let $\phi_{*}^{\prime}$ be the composition

$$
H(A / \pi) \stackrel{\phi_{*}}{\rightarrow} H(A) \rightarrow H(A) / \pi .
$$

Then $\eta_{*}^{\prime} \phi_{*}^{\prime}(x)=n x$ and $\phi_{*}^{\prime} \eta_{*}^{\prime}(y)=n y$. The result follows immediately from this. Namely, if $\eta_{*}^{\prime}(y)=0$ then $n y=\phi_{*}^{\prime} \eta_{*}^{\prime}(y)=0$, while if $x \in H(A / \pi)$ then $n x=\eta_{*}^{\prime} \phi_{*}^{\prime}(x)$ is in the image of $\eta_{*}^{\prime}$.

COROLlaRy 11.1. For any $n, n$ annihilates the kernel and cokernel of $H\left(X^{n}\right) / \pi \rightarrow H\left(C P^{n} X\right)$. Here $\pi$ is the cyclic group described in $\S 5$.

This was originally proved by Richardson [17] in exactly the same way.

REMARK 11.3. Both Theorems 11.1 and 11.2 have obvious duals in cohomology. The dual of Theorem 11.2 can be extended to Čech and AlexanderSpanier cohomology. The resulting theorem generalizes results of Conner [5], Eckmann [9], and Serre [3]. For details see [26].

12. The homology of cyclic products. Let $P \in \odot$. For each prime $p$, we have defined a map $u_{p}: P \rightarrow F_{p}(P)$. Form the direct sum $P+\sum F_{p}(P)$ and define $F(P)$ to be the quotient of this sum obtained by making the identifications $u_{p}(x) \sim x$ for all $x$ in $P$. Now, $\sum F_{p}(P)$ maps onto $F(P)$ under this identification. We filter $F(P)$ by defining $\Phi_{k} F(P)$ to be the image of $\sum \Phi_{k} F_{p}(P)$ in $F(P)$. This makes $F(P)$ an object of the category $\mathcal{F}$.

The injections of $P$ and $F_{p}(P)$ into $P+\sum F_{p}(P)$ induce maps $u: P \rightarrow F(P)$ and $j_{p}: F_{p}(P) \rightarrow F(P)$. Clearly, $u$ is a $P \mathcal{F}$-map and the $j_{p}$ are $\mathscr{F}$-maps. Also $j_{p} u_{p}=u$ for all $p$.

If $f: P \rightarrow P^{\prime}$ in $P$, the maps $F_{p}(f): F_{p}(P) \rightarrow F_{p}\left(P^{\prime}\right)$ were chosen to be $F_{p^{-}}$ 
maps such that $F_{p}(f) u_{p}=u_{p}^{\prime} f$. Therefore, $f+\sum F_{p}(f): P+\sum F_{p}(P) \rightarrow P^{\prime}$ $+\sum F_{p}\left(P^{\prime}\right)$ induces a map $F(f): F(P) \rightarrow F\left(P^{\prime}\right)$ such that $F(f) u=u^{\prime} f$. Suppose we make different choices $F_{p}(f)^{\prime}$ for the $F_{p}(f)$. Then, by the strong homotopy extension property, there are homotopies $S_{p}: F_{p}(f) \simeq F_{p}(f)^{\prime}$ in $F_{p}$ such that $S_{p} u_{p}=0$. Consequently, $0+\sum S_{p}$ induces $S: F(P) \rightarrow F\left(P^{\prime}\right)$ in $\mathcal{F}$ such that $S: F(f) \simeq F(f)^{\prime}$. This implies that $F$ is a prefunctor. Obviously, $u$ is natural and so are the $j_{p}$.

I will now show that $u: P \rightarrow F(P)$ is almost a special $h$-universal $P \mathcal{F}_{\infty}$-map, the only difficulty being that $F(P)$ does not usually lie in $\mathfrak{F}_{\infty}$.

Proposition 12.1. (a) If $f: P \rightarrow A$ is a $\mathrm{PF}_{\infty}$-map, then there is a map $g: F(P) \rightarrow A$ in $\mathcal{F}$ such that $f=g u$.

(b) Let $g_{1}, g_{2}: F(P) \rightarrow A$ be two maps in $\mathcal{F}$ with $A \in \mathcal{F}_{\infty}$. Let $s$ be a PFhomotopy between $g_{1} u$ and $g_{2} u$. Then there is an F-homotopy $S: g_{1} \simeq g_{2}$ such that $S u=s$.

Proof. (a) For each $p, A \in \mathcal{F}_{p}$. Therefore there is a map $g_{p}: F_{p}(P) \rightarrow A$ in $\mathfrak{F}$ such that $g_{p} u=f$. The map $f+\sum g_{p}: P+\sum F_{p}(P) \rightarrow A$ induces the required map g.

(b) For each $p$, consider $g_{i} j_{p}: F_{p}(P) \rightarrow A$. Since $A \in \mathcal{F}_{p}$ and $s: g_{1} j_{p} u_{p} \simeq g_{2} j_{p} u_{p}$, there is an $\mathcal{F}$-homotopy $S_{p}: g_{1} j_{p} \simeq g_{2} j_{p}$ such that $S_{p} u=s$. The homotopy $s+\sum S_{p}: P+\sum F_{p}(P) \rightarrow A$ induces the required homotopy $S$.

Lemma 12.1. For each $p, j_{p}: F_{p}(P) \rightarrow F(P)$ is a monomorphism and $F(P)$ has a $K \pi_{p}$-homotopy, zero on im $j_{p}$, contracting $F(P)$ to $\operatorname{im~} j_{p}$.

Proof. By condition (b) of Theorem 6.1, each $F_{p}(P)$ has a homotopy $S_{p}$ over $K G_{p}$ such that $S_{p}$ is zero on $P$ and contracts $F_{p}(P)$ to $P$. This implies that $P$ is a $K$-direct summand of $F_{p}(P)$. Therefore, $j_{p}$ is a monomorphism.

Now, for $q \neq p, \pi_{p} \subset G_{q}$. Therefore, $S_{q}$ is a $K \pi_{p}$-homotopy for $q \neq p$. Define a homotopy $D$ on $P+\sum F_{q}(P)$ by taking 0 on $P$ and $F_{p}(P)$ and $S_{q}$ on $F_{q}(P)$. Then $D$ induces a $K \pi_{p}$-homotopy on $F(P)$ which contracts it to $\operatorname{im} j_{p}$.

Corollary 12.1. For each $p$,

$$
\left(j_{p} / \pi_{p}\right)_{*}: H\left(F_{p}(P) / \pi_{p}\right) \rightarrow H\left(F(P) / \pi_{p}\right)
$$

is an isomorphism.

We now come to the main theorem. The map $\nabla: \otimes^{n} C(X) \rightarrow C\left(X^{n}\right)$ is a $\odot \mathcal{F}_{\infty}$-map. Therefore, there is an $\mathcal{F}$-map $g: F\left(\otimes^{n} C(X)\right) \rightarrow C\left(X^{n}\right)$, unique up to F-homotopy, such that $\nabla=g u$. The map $g$ is clearly a natural transformation of prefunctors since the maps $g_{p}$ are.

Theorem 12.1. The map

$$
(g / \pi)_{*}: H\left(F\left(\otimes^{n} C(X)\right) / \pi\right) \rightarrow H\left(C P^{n} X\right)
$$

is an isomorphism. 
Proof. By Theorem 11.1, it is sufficient to show that $\left(g / \pi_{p}\right)_{*}$ is an isomorphism for each $p$. Let $g_{p}=g j_{p}$. Then $\left(g_{p} / \pi_{p}\right)_{*}$ is an isomorphism by Theorem 6.2. But, $\left(j_{p} / \pi_{p}\right)_{*}$ is also an isomorphism by Corollary 12.1 .

REMARK 12.1. We can generalize this result as follows. Let $R$ and $\mathcal{C}$ be as in Theorem 11.1. Define $F^{\prime}(P)$ in the same way as $F(P)$ but using only those primes $p$ with $p \in R$. The above arguments now show that $F^{\prime}$ is a prefunctor and

$$
(g / \pi)_{*}: H\left(F^{\prime}\left(\otimes^{n} C(X)\right) / \pi\right) \rightarrow H\left(C P^{n} X\right)
$$

is a $\mathcal{C}$-isomorphism.

If $K$ is a field, we can apply Theorem 13.3 in Chapter $\mathrm{V}$ of [11] to get

Corollary 12.2. If $K$ is a field, $g / \pi$ is a homotopy equivalence.

If $K=Z, F(P) / \pi$ will in general not be free and we cannot apply Theorem 13.3 of [11]. However, the above corollary still holds provided we factor out the 2 -torsion in $F(P) / \pi$.

Proposition 12.2. Assume $K=Z$. Let $E$ be the subcomplex of $F(P) / \pi$ consisting of all elements of order 2 . Then

(a) $(F(P) / \pi) / E$ is $K$-free,

(b) $H(E)=0$.

Corollary 12.3. Let $E$ be as in Proposition 12.2 if $K=Z$ and let $E=0$ if $K$ is a field. Then $\mathrm{g} / \pi$ induces a homotopy equivalence

$$
\left(F\left(\otimes{ }^{n} C(X)\right) / \pi\right) / E \rightarrow C\left(C P^{n} X\right) .
$$

Note that $g / \pi$ maps $E$ to 0 since $C\left(C P^{n} X\right)$ is $K$-free.

In the proof of Proposition 12.2, I will use the following lemma. If $A$ is any abelian group, $A^{(p)}$ will denote its $p$-primary component.

LeMma 12.2. Let $\pi$ be a group of order prime to $p$ which acts on an abelian group $A$. Then $(A / \pi)^{(p)}=A^{(p)} / \pi$.

Proof. We merely apply Richardson's argument (proof of Theorem 11.2) with the functor $H$ replaced by the functor sending $A$ to $A^{(p)}$.

Proof of Proposition 12.2. For each $n$ and each $p$, we have a partial $p$ resolution of $A_{n}$ beginning $\epsilon_{p}: A_{n} \rightarrow W_{n, p}$ say. By Remark 10.3, we can prove assertion (a) by considering $A_{n}+\sum_{p} W_{n, p}$ only. We make the identifications $\boldsymbol{\epsilon}_{p}(a)=a$ for all $p$ and all $a \in A_{n}$ getting a $K \pi$-module $B_{n}$. We must then show that if we take $B / \pi$ and factor out the elements of order 2 , the result is $K$ free. We can reduce this immediately to the case where $A_{n}$ has a single generator $e$. We then take the explicit $W_{n, p}$ given in the proof of Lemma 8.1, and write down generators and relations for $B / \pi$. I will leave this to the reader since it is purely mechanical and quite tedious.

It now remains to prove (b). Let $E^{\prime}$ be the 2-primary component of 
$F(P) / \pi_{2}$. Then $E=E^{\prime} /\left(\pi / \pi_{2}\right)$ by Lemma 12.2 . The fact that $E$ is actually the 2-primary component of $F(P) / \pi$ follows from (a). Now, Lemma 12.1 implies that $F_{2}(P)$ is a $K \pi_{2}$-direct summand of $F(P)$ when considered as a module alone. Therefore we have the split exact sequence

$$
0 \rightarrow F_{2}(P) / \pi_{2} \rightarrow F(P) / \pi_{2} \rightarrow\left(\operatorname{ckr} j_{2}\right) / \pi_{2} \rightarrow 0 .
$$

But, $F_{2}(P) / \pi_{2}$ is $K$-free since $F_{2}(P)$ has a base permuted by $\pi_{2}$ without changes of sign. Since $Z$ has no element of order 2, $E^{\prime}$ maps isomorphically onto the 2-primary component $E^{\prime \prime}$ of $\left(\mathrm{ckr} j_{2}\right) / \pi_{2}$. Now, by Lemma 12.1, ckr $j_{2}$ has a $K \pi_{2}$-homotopy contracting it to 0 . Therefore, $\left(\operatorname{ckr} j_{2}\right) / \pi_{2}$ has a $K$-homotopy contracting it to 0 . The restriction of this homotopy to $E^{\prime \prime}$ has image in $E^{\prime \prime}$ and so contracts $E^{\prime \prime}$ to 0 . Thus $H\left(E^{\prime}\right)=H\left(E^{\prime \prime}\right)=0$.

By Theorem 11.2, $H(E)=H\left(E^{\prime}\right) /\left(\pi / \pi_{2}\right)$ since $H(E)$ and $H\left(E^{\prime}\right)$ are 2primary while $\pi / \pi_{2}$ has odd order. This shows that $H(E)=0$.

\section{Preservation of homotopy.}

Definition 13.1. We say that a prefunctor $T$ is additive if $T(f+g)$ $\simeq T(f)+T(g)$ for all $f$ and $g$.

The prefunctors $F_{p}$ are additive because $F_{p}(f+g) u_{p}=F_{p}(f) u_{p}+F_{p}(g) u_{p}$ and $u_{p}$ is $h$-universal. It is easy to show that $F$ is also additive by using the strong homotopy extension property. We merely choose homotopies $S_{p}: F_{p}(f+g) \simeq F_{p}(f)+F_{p}(g)$ such that $S_{p} u_{p}=0$. These $S_{p}$ then induce the required homotopy $S$ between $F(f+g)$ and $F(f)+F(g)$.

The following lemma holds not only for cyclic $\pi$ but for any subgroup of the symmetric group on $n$ elements provided we redefine the category $\mathbb{P}_{0}$ in the appropriate way. I will leave this generalization to the reader.

Lemma 13.1. Let $T$ be a covariant prefunctor from the category $\odot$ to the category of chain complexes, chain maps, and chain homotopies over $K$. Define a prefunctor $S$ on the category of $K$-free chain complexes by $S(M)=T\left(\otimes^{n} M\right)$ with obvious induced maps. Assume $T$ is additive and that $H(P)=0$ implies $H(T(P))$ $=0$ for any $P \in \mathcal{P}_{0}$. Then

(a) $f_{0} \simeq f_{1}: M \rightarrow M^{\prime}$ implies

$$
S\left(f_{0}\right)_{*}=S\left(f_{1}\right)_{*}: H(S(M)) \rightarrow H\left(S\left(M^{\prime}\right)\right) .
$$

(b) If $T(P)$ is $K$-free for all $P \in \mathcal{P}_{0}$, then the prefunctor $S$ preserves homotopy.

Proof. Let $I$ be the chain complex having two generators $a$ and $b$ in dimension 0 , and one generator $e$ in dimension 1 with $\partial e=a-b$. Define $i_{0}, i_{1}: K \rightarrow I$ by $i_{0}(1)=a$ and $i_{1}(1)=b$. Two maps $f_{0}, f_{1}: M \rightarrow M^{\prime}$ are homotopic if and only if there is a map $g: M \otimes I \rightarrow M^{\prime}$ such that $f_{0}=g\left(1 \otimes i_{0}\right)$ and $f_{1}$ $=g\left(1 \otimes i_{1}\right)$. Consequently, it is sufficient to prove the lemma in the case where $M^{\prime}=M \otimes I$ and $f_{j}=1 \otimes i_{j}$ for $j=1,2$.

Let $I=K+I^{*}$ where $K$ is identified with $K a$ and $I^{*}$ is generated by $e$ and 
$a-b$. Then $H\left(I^{*}\right)=0$ while $i_{0}$ and $i_{1}$ have the same projection on the summand $K$. This decomposition leads to a decomposition $M \otimes I=M+M^{*}$ where $M^{*}=M \otimes I^{*}$. Again $H\left(M^{*}\right)=0$ while $f_{0}$ and $f_{1}$ have the same projection on the summand $M$.

Now, $\otimes^{n}(M \otimes I)=\otimes^{n}\left(M+M^{*}\right)=\otimes^{n} M+P$ where $P$ consists of all terms with a factor $M^{*}$. Choose $K$-bases for $M$ and $M^{*}$. These together form a $K$ base $\left\{e_{i}\right\}$ for $M+M^{*}$. A $K$-base for $P$ is then given by all products $e_{i_{1}} \otimes \cdots \otimes e_{i_{n}}$ with at least one $e_{i_{\nu}} \in M^{*}$. This base is permuted by $\pi$ up to sign. It is now trivial to verify that $P \in \mathcal{P}_{0}$, the argument being the same as the one which shows that $\otimes^{n} M \in \mathcal{P}_{0}$ for any $K$-free mdule $M$. As before, $H(P)=0$ while $\otimes^{n} f_{0}$ and $\otimes^{n} f_{1}$ have the same projection on the summand $\otimes{ }^{n} M$. Therefore $\otimes{ }^{n} f_{0}-\otimes{ }^{n} f_{1}$ factors through $P$. Consequently, $S\left(f_{0}\right)-S\left(f_{1}\right)$ is homotopic to $T\left(\otimes^{n} f_{0}-\otimes^{n} f_{1}\right)$ which factors through $T(P)$. But, $H(T(P))$ $=0$. This proves $(\mathrm{a})$.

If, in addition, $T(P)$ is $K$-free, then $H(T(P))=0$ implies $T(P) \simeq 0$. Therefore $S\left(f_{0}\right) \simeq S\left(f_{1}\right)$. This proves (b).

Remark 13.1. Suppose $X$ is a free acyclic $K \pi$-complex. Then the lemma, applied to the functor $P \otimes_{\pi} X$ gives another proof of Theorem 3.1 of [22].

REMARK 13.2. The lemma may be generalized by letting $\mathcal{e}$ be a Serre class of modules and assuming that $H(P)=0$ implies $H(T(P)) \in \mathcal{C}$. The conclusion then becomes $S\left(f_{0}\right)_{*} \equiv S\left(f_{1}\right)_{*} \bmod \mathcal{C}$, i.e. their difference factors through a module in $\mathcal{C}$. The proof is exactly the same.

Lemma 13.2. Let $P \in \mathcal{P}_{0}$ be such that $H(P)=0$. Then $H(F(P) / \pi)=0$.

Proof. By Lemma 6.1, $H\left(F_{p}(P) / \pi_{p}\right)=0$ for all $p$. By Corollary 12.1, $H\left(F(P) / \pi_{p}\right)=0$. The result now follows from Theorem 11.1 applied to the map $F(P) \rightarrow 0$.

We now define a prefunctor $S$ from $K$-free chain complexes to $K$-free chain complexes by $S(M)=\left(F\left(\otimes^{n} M\right) / \pi\right) / E$ where $E$ is as in Corollary 12.3. The map $g: F\left(\otimes^{n} C(X)\right) \rightarrow C\left(X^{n}\right)$ induces a map $h: S(C(X)) \rightarrow C\left(C P^{n} X\right)$ which is a homotopy equivalence by Corollary 13.3.

Theorem 13.1. The prefunctor $S$ preserves homotopy.

Proof. This is an immediate consequence of Lemma 13.1, Lemma 13.2, and Proposition 12.2.

Corollary 13.1. In computing $H\left(C P^{n} X\right)$ as $H(S(C(X)))$ we may replace $C(X)$ by any $K$-free chain complex $M$ having the same homotopy type as $C(X)$. In particular, if $H(X)$ is $K$-free, then $H\left(C P^{n} X\right)=H(S(H(X)))$.

14. Products. If $\pi$ acts on two modules $A$ and $B$, we make $\pi$ act on $A \otimes B$ by $t(a \otimes b)=t a \otimes t b$ (i.e. by composing the diagonal map $\pi \rightarrow \pi \times \pi$ with the obvious action of $\pi \times \pi$ on $A \otimes B$ ). If $A, B \in \mathcal{F}$, we filter $A \otimes B$ by

$$
\Phi_{k}(\mathrm{~A} \otimes B)=\sum \Phi_{r}(A) \otimes \Phi_{s}(B)
$$


the sum being over all $(r, s)$ such that $r+s=k$. If $A$ and $B$ are $K$-free, a base for $A \otimes B$ is obtained by taking products of basis elements for $A$ and $B$. An element $e_{i} \otimes e_{j}^{\prime}$ of this base is fixed under $t \in \pi$ if and only if both $e_{i}$ and $e_{j}^{\prime}$ are fixed under $t$. Thus we see easily that $A \otimes B \in \mathcal{F}_{p}$ if $A$ and $B$ are in $\mathcal{F}_{p}$.

Now, let $P_{1}, P_{2} \in \mathcal{P}$. Clearly $P_{1} \otimes P_{2} \in \mathcal{P}$. Consider $u_{p} \otimes u_{p}: P_{1} \otimes P_{2} \rightarrow F_{p}\left(P_{1}\right)$ $\otimes F_{p}\left(P_{2}\right)$. This is a $\odot \mathcal{F F}_{p}$-map and so there is a factorization through $u_{p}: P_{1}$ $\otimes P_{2} \rightarrow F_{p}\left(P_{1} \otimes P_{2}\right)$. This gives us a map $F_{p}\left(P_{1} \otimes P_{2}\right) \rightarrow F_{p}\left(P_{1}\right) \otimes F_{p}\left(P_{2}\right)$.

The compositions

$$
F_{p}\left(P_{1} \otimes P_{2}\right) \rightarrow F_{p}\left(P_{1}\right) \otimes F_{p}\left(P_{2}\right) \rightarrow F\left(P_{1}\right) \otimes F\left(P_{2}\right)
$$

agree on $P_{1} \otimes P_{2}$ and so induce a map $F\left(P_{1} \otimes P_{2}\right) \rightarrow F\left(P_{1}\right) \otimes F\left(P_{2}\right)$. It is easy to verify that this is a natural transformation of prefunctors. This map induces a map

$$
F\left(P_{1} \otimes P_{2}\right) / \pi \rightarrow F\left(P_{1}\right) / \pi \otimes F\left(P_{2}\right) / \pi
$$

and a map

$$
\left(F\left(P_{1} \otimes P_{2}\right) / \pi\right) / E \rightarrow\left(F\left(P_{1}\right) / \pi\right) / E \otimes\left(F\left(P_{2}\right) / \pi\right) / E .
$$

If $M$ and $N$ are chain complexes, there is a natural isomorphism $\otimes^{n}(M \otimes N)$ $\rightarrow \otimes^{n} M \otimes \otimes^{n} N$ given by

$$
x_{1} \otimes y_{1} \otimes \cdots \otimes x_{n} \otimes y_{n} \rightarrow \pm\left(x_{1} \otimes \cdots \otimes x_{n}\right) \otimes\left(y_{1} \otimes \cdots \otimes y_{n}\right),
$$

the sign being the usual one associated with a permutation of elements in graded modules. Combining this map with the one considered above, we get a map

$$
S(M \otimes N) \rightarrow S(M) \otimes S(N)
$$

where $S$ is as in Theorem 13.1 .

If $X$ and $Y$ are semisimplicial complexes, there is an obvious isomorphism $(X \times Y)^{n} \rightarrow X^{n} \times Y^{n}$ given by

$$
\left(x_{1}, y_{1}, \cdots, x_{n}, y_{n}\right) \rightarrow\left(x_{1}, \cdots, x_{n}, y_{1}, \cdots, y_{n}\right) .
$$

This induces a map

$$
C P^{n}(X \times Y) \rightarrow C P^{n} X \times C P^{n} Y .
$$

This, of course, is not an isomorphism in general.

Let $f: C(X \times Y) \rightarrow C(X) \otimes C(Y)$ be the "Alexander-Čech-Whitney" map i.e. the map $f$ of $\left[10\right.$, Chapter I, §2]. Define maps $\phi^{\prime}$, and $\phi^{\prime \prime}$ to be the following compositions.

$$
\begin{gathered}
\phi^{\prime}: \otimes^{n} C(X \times Y) \stackrel{\otimes^{n}}{\longrightarrow} \otimes^{n}(C(X) \otimes C(Y)) \rightarrow \otimes^{n} C(X) \otimes \otimes^{n} C(Y), \\
\phi^{\prime \prime}: C\left(C P^{n}(X \times Y)\right) \longrightarrow C\left(C P^{n} X \times C P^{n} Y\right) \stackrel{f}{\rightarrow} C\left(C P^{n} X\right) \otimes C\left(C P^{n} Y\right) .
\end{gathered}
$$


We can apply $F$ to $\phi^{\prime}$ and reduce by $\pi$, getting a map

$$
\phi_{0}^{\prime}: S(C(X \times Y)) \rightarrow S(C(X)) \otimes S(C(Y)) .
$$

Proposition 14.1. The following diagram commutes up to homotopy

$$
\begin{array}{cc}
S(C(X \times Y)) \stackrel{\phi_{0}^{\prime}}{\longrightarrow} S(C(X)) \otimes S(C(Y)) \\
\downarrow g / \pi & \downarrow g / \pi \otimes g / \pi \\
C\left(C P^{n}(X \times Y)\right) \stackrel{\phi^{\prime \prime}}{\longrightarrow} C\left(C P^{n} X\right) \otimes C\left(C P^{n} Y\right) .
\end{array}
$$

Proof. The maps $\nabla$ and $f$ between $C(X \times Y)$ and $C(X) \otimes C(Y)$ are homotopy inverses. Since $S$ preserves homotopy, it is sufficient to verify that

$$
\phi^{\prime \prime} g / \pi \circ S(\nabla) \simeq(g / \pi \otimes g / \pi) \phi_{0}^{\prime} \circ S(\nabla) .
$$

Because of the $h$-universal property, it is now sufficient to check that the following diagram commutes.

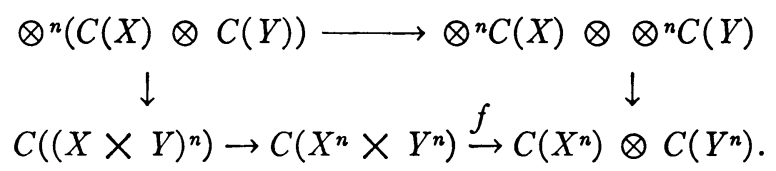

To do this, note that if $f$ is replaced by $\nabla$ in this diagram (reversing the arrow), the resulting diagram involves only Eilenberg-Zilber maps and so will commute. The desired result then follows from the well known relation $f \nabla=$ identity [10, Chapter I, $\S 2]$.

Consider now the diagonal map $X \rightarrow X \times X$. This, combined with the map $f$, gives us the usual diagonal chain map $d: C(X) \rightarrow C(X) \otimes C(X)$. By applying $S$, we get

$$
S(C(X)) \rightarrow S(C(X)) \otimes S(C(X)) .
$$

TheOREM 14.1. The following diagram commutes up to homotopy.

$$
\begin{array}{ccc}
S(C(X)) \rightarrow S(C(X)) \otimes S(C(X)) & \\
\downarrow & \downarrow g / \pi & \downarrow \\
C\left(C P^{n} X\right) & \stackrel{d}{\rightarrow} C\left(C P^{n} X\right) \otimes C\left(C P^{n} X\right) .
\end{array}
$$

Proof. This follows immediately from Proposition 14.1, the naturality of $g$, and the fact that the diagonal map for $C P^{n} X$ is the composition

$$
C P^{n} X \rightarrow C P^{n}(X \times X) \rightarrow C P^{n} X \times C P^{n} X .
$$

This theorem shows that if we know a diagonal chain map for $X$, we can find one for $C P^{n} X$. If, in computing, we replace $C(X)$ by a small $K$-free chain complex $M \simeq C(X)$, we need only find a map $d: M \rightarrow M \otimes M$ such that the diagram 


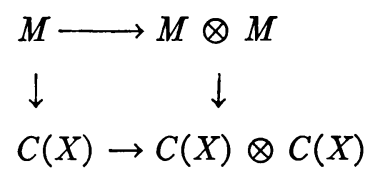

commutes up to homotopy.

15. Some spectral sequences. The proofs of Theorem 6.2 and Lemma 6.1 depend on the use of some comparison theorems. Borrowing some terminology of J. H. C. Whitehead, I will divide these theorems into crude and delicate comparison theorems. These theorems in turn depend on the use of some spectral sequences.

The first of these sequences is defined for all groups $\pi$ and is merely the well known sequence of Cartan and Leray [3] and [2, Chapter XVI, Theorem 8.4] but without any assumptions of freeness or weak projectivity. This sequence is defined as follows. Let $X$ be a free acyclic $K \pi$-complex. Let $A$ be a chain complex over $K \pi$. Consider the double complex $X \otimes_{\pi} A$. There are two associated spectral sequences $[2$, Chapter $\mathrm{XV}, \S 6]$. I will denote these by $I$ and $I I$ as in [2], and by $I(A)$ and $I I(A)$ if it is necessary to specify $A$. The first of these sequences has $I_{r, s}^{2}=H_{r}\left(\pi, H_{s}(A)\right)$, and $I^{\infty}$ is a graded module obtained from the first filtration of $H\left(X \otimes_{\pi} A\right)$. Note that we cannot say that this is $H(A / \pi)$ because $A$ is not assumed weakly projective. The second spectral sequence has $I I_{r, s}^{2}=H_{s}\left(H_{r}(\pi, A)\right)$. Note that I am not permuting indices as in [2, Chapter XV, §6]. Here, of course, $H_{r}(\pi, A)$ for each $r$ is a chain complex with boundary operator induced by that of $A$. The term $I I^{\infty}$ is the graded module obtained from the second filtration of $H\left(X \otimes_{\pi} A\right)$. The only use I will make here of these sequences is in the proof of the following theorem which holds for all groups $\pi$.

ThEOREM 15.1 (The CRUde COMPARISON THEOREM). Let $\mathfrak{C}$ be a Serre class of modules. If $\pi$ is infinite, let $\mathfrak{e}$ satisfy axiom $I I_{B}$ of [20]. Let $A$ and $B$ be chain complexes over $K \pi$. Let $f: A \rightarrow B$ be a chain map over $K \pi$. Assume that

(a) $f_{*}: H(A) \rightarrow H(B)$ is a e-isomorphism.

(b) For $r>0, f_{* *}: H\left(H_{r}(\pi, A)\right) \rightarrow H\left(H_{r}(\pi, B)\right)$ is a e-isomorphism.

Then $f_{*}: H(A / \pi) \rightarrow H(B / \pi)$ is also a e-isomorphism.

Proof. Let $C$ be the relative mapping cylinder [4, Exp. 3] [15, Chapter IV] of $f: A \rightarrow B$. The mapping cylinder is formed by a direct sum construction and so is preserved by all additive functors. Therefore, $H_{r}(\pi, C)$ is the relative mapping cylinder of $f_{*}: H_{r}(\pi, A) \rightarrow H_{r}(\pi, B)$ and $C / \pi$ is the relative mapping cylinder of $f / \pi: A / \pi \rightarrow B / \pi$.

Consider the spectral sequences of $C$. By (a), $H(C) \equiv 0 \bmod \mathfrak{e}$ and so $I^{2}$ $\equiv 0 \bmod \mathcal{C}$. This implies that $H\left(X \otimes_{\pi} C\right) \equiv 0 \bmod \mathcal{C} . \mathrm{By}(\mathrm{b}), I I_{r, s}^{2}=H_{s}\left(H_{r}(\pi, C)\right)$ $\equiv 0 \bmod \mathfrak{C}$ for $r \neq 0$. Therefore $I I^{2} \equiv I I^{\infty}$. But $I I^{\infty} \equiv 0 \bmod \mathcal{C}$. Thus $I I^{2}$ $\equiv 0 \bmod \mathcal{C}$. In particular, 


$$
I I_{0, s}^{2}=H_{s}\left(H_{0}(\pi, C)\right)=H_{s}(C / \pi) \equiv 0 \bmod \mathcal{C} .
$$

This immediately implies the desired result.

The theorem and the spectral sequence have obvious duals in cohomology (using $\operatorname{Hom}_{\pi}(X, A)$ ).

The delicate comparison theorems will be given in $\S 17$. Their proof depends on the use of spectral sequences analogous to those above but using the Tate cohomology theory [2, Chapter XII] in place of the usual cohomology theory of groups.

Let $\pi$ be any finite group and let $X$ be a Tate complex (or complete resolution in the terminology of [2, Chapter XII, §3]) for $\pi$. We again consider $X \otimes_{\pi} A$ for any chain complex $A$ over $K \pi$. This again has two associated spectral sequences. The first of these always converges [2, Chapter XV, §6] while the second converges provided $A$ is finite dimensional. The terms $I^{\infty}$ and $I I^{\infty}$ are obtained from the first and second filtrations of $H\left(X \otimes_{\pi} A\right)$. The initial terms of the sequences are $I_{r, s}^{2}=\hat{H}_{r}\left(\pi, H_{s}(A)\right)$ and $I I_{r, s}^{2}=H_{s}\left(\hat{H}_{r}(\pi, A)\right)$. Here, for any $K \pi$-module $M, \hat{H}_{r}(\pi, M)=H_{r}\left(X \otimes_{\pi} M\right)$ is the $r$ th Tate homology module of $M$. These have the following properties.

(1) If $0 \rightarrow M^{\prime} \rightarrow M \rightarrow M^{\prime \prime} \rightarrow 0$ is exact, there is a natural exact sequence

$$
\rightarrow \hat{H}_{r}\left(\pi, M^{\prime}\right) \rightarrow \hat{H}_{r}(\pi, M) \rightarrow \hat{H}_{r}\left(\pi, M^{\prime \prime}\right) \rightarrow \hat{H}_{r-1}\left(\pi, M^{\prime}\right) \rightarrow \text {. }
$$

(2) If $M$ is weakly projective, $\hat{H}_{r}(\pi, M)=0$ for all $r$ (even $r=0$ ). For example, this holds if $M$ is free.

(3) If $r>0, \hat{H}_{r}(\pi, M)=H_{r}(\pi, M)$, the ordinary homology module. This isomorphism is natural.

The proofs of these results may easily be obtained by dualizing the proofs of the corresponding results for Tate cohomology [2, Chapter XII]. It can easily be shown that these modules and the spectral sequences are independent of the choice of $X$. However, I shall be concerned here only with the case in which some definite $X$ has been chosen.

I will need one further property of these spectral sequences.

Lemma 15.1. Let $A$ be a finite dimensional chain complex over $K \pi$. Assume that $A$ has a $K$-free subcomplex $L$ on which $\pi$ acts trivially such that $A / L$ is weakly projective as a $K \pi$-module. Then, in the spectral sequences just described, $I I^{2}=I I^{\infty}$ and $i_{* *}: I I^{2}(L) \approx I I^{2}(A)$.

Proof. Consider the exact sequence

$$
0 \rightarrow L \rightarrow A \rightarrow A / L \rightarrow 0 .
$$

By property (1) of the Tate homology groups, we get an exact sequence of homology groups. By property $(2), \hat{H}_{r}(\pi, A / L)=0$ for all $r$. Therefore the exact sequence gives us the isomorphism $i_{*}: \hat{H}_{r}(\pi, L) \rightarrow \hat{H}_{r}(\pi, A)$. Passing to homology, we see that $i_{* *}: I I^{2}(L) \rightarrow I I^{2}(A)$ is an isomorphism. Therefore, the 
second spectral sequences of $L$ and $A$ are isomorphic. This includes the terms $I I^{\infty}$ since the finite dimensionality of $A$ (and so of $L$ ) insures that the second spectral sequences converge. Consequently, it is sufficient to show that $I I^{2}(L)=I I^{\infty}(L)$.

Now, $\pi$ acts trivially on $L$. Since $L$ is $K$-free and $K$ is hereditary, we can split $L$ over $K$ (and so over $K \pi$ ) into a direct sum $\sum L^{(i)}$ where each $L^{(i)}$ is a chain complex zero except in dimensions $i$ and $i+1$ (cf. [11, Chapter $V$, $\S 8])$. Consequently, the spectral sequences for $L$ are the direct sums of the spectral sequences for the $L^{(i)}$. However, in the sequence for $L^{(i)}$, we have $I I^{2}=I I^{\infty}$ because $I I_{r, s}^{2}=0$ unless $q=i$ or $i+1$ while $\operatorname{deg} d^{k}=(k-1,-k)$.

Note that the result may be false if $L$ is not $K$-free. For example, take $K=Z, \pi=Z_{2}$, and let $L$ be $0 \rightarrow Z \rightarrow^{2} Z \rightarrow Z_{2} \rightarrow 0$ with $A=L$. The example with $A$ equal to the acyclic complex $0 \rightarrow K \rightarrow K \pi \rightarrow K \pi \rightarrow K \rightarrow 0$ shows that it is necessary to assume that $L$ is a subcomplex.

The next lemma is due essentially to Smith [21] if $\pi$ is cyclic.

Lemma 15.2. Let $\mathfrak{e}$ be a Serre class of modules. Let $A$ satisfy the conditions of Lemma 15.1. Let $H(A) \equiv 0 \bmod \mathcal{C}$. Then $H(A / \pi) \equiv 0 \bmod \mathfrak{e}$ and $H\left(\hat{H}_{r}(\pi, A)\right)$ $\equiv 0 \bmod \mathcal{e}$ for all $r$.

Proof. In the first spectral sequence, we have $I_{r, s}^{2}=\hat{H}_{r}\left(\pi, H_{s}(A)\right) \equiv 0 \bmod \mathrm{C}$. Therefore, the limit term is in $\mathcal{C}$ and so $I I^{\infty} \equiv 0 \bmod \mathcal{C}$. By Lemma 15.1, $I I_{r, s}^{2}=H_{s}\left(\hat{H}_{r}(\pi, A)\right) \equiv 0 \bmod \mathcal{C}$. Since $\hat{H}_{r}(\pi, A)=H_{r}(\pi, A)$ for $r>0$, the conditions of Theorem 15.1 are satisfied by the map $A \rightarrow 0$. Therefore $H(A / \pi)$ $\equiv 0 \bmod$ e.

REMARK 15.1. Everything in this section dualizes to cohomology. In this form, the theory can be applied to the cohomology theory of spaces with finite groups of transformations. In this way, we can get new proofs and generalizations of the well known theorems of Smith, Floyd, and Liao. See [24] for details.

16. Proof of Lemma 6.1. I will prove the following stronger result.

Lemma 16.1. Let $\mathfrak{e}$ be a Serre class of modules. Let $P \in \mathcal{P}_{0}$ be such that $H(P) \equiv 0 \bmod$ e. Then $H\left(F_{p}(P) / \pi_{p}\right) \equiv 0 \bmod$ e.

Proof. By Remark 6.1, it is sufficient to consider the case where $\pi=\pi_{p}$. Assume that the lemma has been proved for cyclic $p$-groups of order less than that of $\pi$. It is certainly true if $\pi$ has order 1 since we can then choose $F_{p}(P)$ $=P$. Thus, if $\pi^{\prime}$ is the subgroup of index $p$ in $\pi$, we can assume that $H\left(F_{p}(P) / \pi^{\prime}\right) \equiv 0 \bmod \mathfrak{e}$ whenever $H(P) \equiv 0 \bmod \mathcal{e}$. This uses the inductive assumption and Remark 10.1.

In (a) of Definition 6.5, the complexes $M_{i}$ are $K$-free. As in the proof of Lemma 15.1, we can split them into direct sums of complexes each zero except in two consecutive dimensions. If we multiply out the resulting expression for the object of $P$, we see that it is a possibly infinite direct sum of expres- 
sions of the form (a) where each $M_{i}$ is zero except in two consecutive dimensions (depending on $i$ ).

Now, if $P=\sum P^{(k)}$ and $H(P) \equiv 0$, then $H\left(P^{(k)}\right) \equiv 0$ for each $k$. We can choose $F_{p}(P)=\sum F_{p}\left(P^{(k)}\right)$ since the universal mapping property will clearly be satisfied. In fact, the construction of $F_{p}(P)$ will give this result automatically if we take as base for $P$ the union of bases for the $P^{(k)}$. Furthermore, the decomposition of the $M_{i}$ was such that only a finite number of the $P^{(k)}$ will have nonzero components below any given dimension. By Remark 10.2, we may choose the $F_{p}\left(P^{(k)}\right)$ to have the same property. Therefore, each direct sum

$$
H_{r}\left(F_{p}(P)\right)=\sum H_{r}\left(F_{p}\left(P^{(k)}\right)\right)
$$

has only a finite number of nonzero terms. Consequently, it is sufficient to prove Lemma 6.1 when $P$ is an expression of type (a) (in Definition 6.5) with each $M_{i}$ being zero except in two consecutive dimensions (depending on $i$ ). Such a complex $P$ is clearly finite dimensional so we may assume $F_{p}(P)$ is finite dimensional by Remark 10.4.

There are two cases to consider depending on the integer $r$ in Definition 6.5 .

CASE 1. $r \neq 1$. In this case, no basis element of $P$ is fixed up to sign under the generator $T$ of $\pi$. Consequently, by Remarks 8.5 and 10.3 , the same is true of $F_{p}(P)$. Since $\pi / \pi^{\prime}$ is cyclic of prime order $p, F_{p}(P) / \pi^{\prime}$ must be $\pi / \pi^{\prime}$ free. Therefore Lemma 15.2 applies (with $\pi / \pi^{\prime}$ in place of $\pi$ ) since we can take $L=0$. This shows that

$$
H\left(F_{p}(P) / \pi\right)=H\left(\left(F_{p}(P) / \pi^{\prime}\right) /\left(\pi / \pi^{\prime}\right)\right) \equiv 0 \bmod \mathfrak{C} .
$$

CASE 2. $r=1$. The complex $P$ now has the form $P=\otimes^{n} M$ where $M$ is zero except in two consecutive dimensions, say $m$ and $m+1$. The only basis elements in $P$ fixed up to sign under the generator $T$ occur in $P_{n m}=\otimes^{n} M_{m}$ and in $P_{n(m+1)}=\otimes^{n} M_{m+1}$. Therefore, the only basis elements of the $W_{s}^{(k)}$ fixed under $T$ are those in $W_{m}^{(n m)}$ and in $W_{m+1}^{(n(m+1))}$. This follows from Remark 8.5, Definition 8.1, and the fact that $W_{s}^{(k)}=0$ for $s<k / p^{h}$ (by Lemma 8.1). But, the only elements of dimension $\leqq m$ in $F_{p}(P)$ are those of $W_{m}^{(n m)}$. Consequently, $W_{m+1}^{(n m+m)}$ and $W_{m}^{(n m)}$ together form a subcomplex of $F_{p}(P)$. Let $L$ be the image of this subcomplex in $F_{p}(P) / \pi^{\prime}$. Then $\pi / \pi^{\prime}$ acts trivially on $L$ since $\pi$ acts trivially on $W_{m+1}^{(n m+m)}$ and $W_{m}^{(n m)}$. In every other $W_{s}^{(k)}$, no basis element is fixed under $T$. As in Case 1 , this shows that $\left(F_{p}(P) / \pi^{\prime}\right) / L$ is $\pi / \pi^{\prime}$ free. Thus we can again apply Lemma 15.2 which shows that

$$
H\left(F_{p}(P) / \pi\right)=H\left(\left(F_{p}(P) / \pi^{\prime}\right) /\left(\pi / \pi^{\prime}\right)\right) \equiv 0 \bmod \mathcal{e} .
$$

REMARK 16.1. Lemma 15.2 yields the further result that $P \in P_{0}$ and $H(P) \equiv 0 \bmod \mathfrak{e}$ imply $H\left(\hat{H}\left(\pi / \pi^{\prime}, F_{p}(P) / \pi^{\prime}\right)\right) \equiv 0 \bmod \mathfrak{e}$. 
REMARK 16.2. We have incidentally shown that for each finite dimensional $P$ in $P_{0}, F_{p}(P) / \pi^{\prime}$ satisfies the conditions of Lemma 15.1 because a finite direct sum of complexes satisfying these conditions will again satisfy these conditions.

In order to avoid repeating the construction used in this section, I will prove now a lemma which will be used in $\S 18$. The lemma will be stated and proved for $K=Z$. If $K$ is a field, the order (number of elements) of a $K$-module should be replaced by its dimension over $K$.

Lemma 16.2. Let $M$ be a finite dimensional, finitely generated $K$-free chain complex. Then,

$$
\text { order } H_{8}\left(\hat{H}_{r}\left(\pi / \pi^{\prime}, F\left(\otimes^{n} M\right) / \pi^{\prime}\right)\right) \leqq \operatorname{order} H_{8}\left(\hat{H}_{r}\left(\pi / \pi^{\prime}, M\right)\right)
$$

where we make $\pi / \pi^{\prime}$ act trivially on $M$ to find $\hat{H}_{r}\left(\pi / \pi^{\prime}, M\right)$.

Proof. Since $M$ is finitely generated, we can find a canonical base for $M$ as in [11, Chapter $\mathrm{V}, \S 8$, Theorem 8.2]. In this way, we split $M$ into the direct sum of elementary complexes $M_{i}$ of the following types.

(1) $M_{i}$ has a single generator $a$ in dimension $m$ and is zero in other dimensions.

(2) $M_{i}$ has one generator $a$ in dimension $m+1$, one generator $b$ in dimension $m$, and is zero in other dimensions. Furthermore, $\partial a=\theta b$ with $\theta \neq 0$.

If we expand $\otimes^{n} M=\otimes^{n}\left(\sum M_{i}\right)$ as in the above proof of Lemma 16.1, we get $\otimes^{n} M=\sum \otimes^{n} M_{i}+P$ where $P$ is the sum of all cross products. Now, in the course of proving Lemma 16.1 , we showed that $F(P) / \pi^{\prime}$ was $\pi / \pi^{\prime}$-free. Therefore,

$$
\hat{H}_{r}\left(\pi / \pi^{\prime}, F\left(\otimes^{n} M\right) / \pi^{\prime}\right) \approx \sum \hat{H}_{r}\left(\pi / \pi^{\prime}, F\left(\otimes^{n} M_{i}\right) / \pi^{\prime}\right) .
$$

Consequently, it is sufficient to prove the lemma when $M$ is elementary.

By Lemma 15.1,

$$
H_{s}\left(\hat{H}_{r}\left(\pi / \pi^{\prime}, F\left(\otimes^{n} M\right) / \pi^{\prime}\right)\right) \approx H_{s}\left(\hat{H}_{r}\left(\pi / \pi^{\prime}, L\right)\right)
$$

since these terms are just the terms $I I^{2}$ of the spectral sequences. Here, of course, we take $L$ as in the proof of Lemma 16.1 to consist of the two submodules $W_{m+1}^{(n m+n)}$ and $W_{m}^{(n m)}$. Now, inspection of the proof of Lemma 8.1 shows that if $M$ is of type (1) then $W_{m+1}^{(n m+n)}=0$ and $W_{m}^{(n m)} \approx K$, while if $M$ is of type (2) then $W_{m+1}^{(n m+n)} \approx K$ and $W_{m}^{(n m)} \approx K$. The group $\pi$ acts trivially on $K$ in all cases. Thus, in both cases, $L \approx M$ as a module although it can and usually will have a different boundary operator. Therefore $\hat{H}_{r}\left(\pi / \pi^{\prime}, L\right)$ and $\hat{H}_{r}\left(\pi / \pi^{\prime}, M\right)$ are also isomorphic as modules. If $M$ is of type (1), the boundary operator is zero and the result follows immediately. Note that $\hat{H}_{r}\left(\pi / \pi^{\prime}, K\right)$ is either $K / p K$ or ${ }_{p} K$ depending on $r$. Therefore, it is finite.

Assume now that $M$ is of type (2) with $\partial a=\theta b$. Every element in $\hat{H}_{r}\left(\pi / \pi^{\prime}, M\right)$ has order $p$ since $\pi / \pi^{\prime}$ has order $p$. Therefore, if $p \mid \theta$, the bound- 
ary operator in $\hat{H}_{r}\left(\pi / \pi^{\prime}, M\right)$ is zero. The desired inequality follows immediately.

There remains the case in which $p \nmid \theta$. In this case, let $\mathbb{e}$ be the Serre class of torsion groups with no $p$-torsion. Then $H(M)$ is in $\mathfrak{e}$ and so $H\left(\otimes^{n} M\right)$ is in e. By Remark 16.1, it follows that $H_{s}\left(\hat{H}_{r}\left(\pi / \pi^{\prime}, L\right)\right)$ is in e. However, every element of this module has order $p$. Therefore, $H_{s}\left(\hat{H}_{r}\left(\pi / \pi^{\prime}, L\right)\right)=0$ in this case.

17. The delicate comparison theorems. The theorems of this section give sufficient conditions for a map $f: A \rightarrow B$ to satisfy the condition (b) of Theorem 15.1. Since $H_{r}(\pi, M)=\hat{H}_{r}(\pi, M)$ for $r>0$ and every $M$, it is sufficient to find conditions under which $f_{* *}: H\left(\hat{H}_{r}(\pi, A)\right) \rightarrow H\left(\hat{H}_{r}(\pi, B)\right)$ is an isomorphism for all $r$. In the case in which these theorems will be applied, $\pi$ will be a cyclic p-group and so all $\hat{H}_{r}(\pi, M)$ will be $p$-groups. Thus there will be no need to state the theorems in terms of $\mathbb{e}$-theory. Throughout this section, however, $\pi$ may be any finite group.

The theorems are based on some simple "pigeon hole" type arguments. If $R$ is a filtered module, I will denote the associated graded module by $\operatorname{Gr}(R)$. Thus $\operatorname{Gr}_{s}(R)=F_{s} R / F_{s-1} R$. I will say that $R$ is bounded below if $F_{8} R=0$ for $-s$ large and that $R$ is bounded above if $F_{8} R=0$ for $s$ large. Note that a filtration preserving map $f: R \rightarrow S$ may be a module isomorphism without being an isomorphism of filtered modules i.e. $f: F_{s} R \rightarrow F_{s} S$ may not be an isomorphism for all $s$. (It is clearly a monomorphism if $f$ is.)

Lemma 17.1. Let $R$ and $S$ be filtered modules which are bounded above. Let $f: R \rightarrow S$ be a filtration preserving map which is a module isomorphism. Assume that for every $s$,

$$
\text { order } \mathrm{Gr}_{s}(R) \leqq \text { order } \mathrm{Gr}_{s}(S)<\infty .
$$

Then $f$ is an isomorphism of filtered modules and consequently, $\operatorname{Gr}(f): \operatorname{Gr}(R)$ $\rightarrow \mathrm{Gr}(S)$ is an isomorphism.

If $K$ is a field, "order" should be replaced by "dimension."

Proof. For large $s, f_{s}: F_{s} R \rightarrow F_{s} S$ is an isomorphism since $R$ and $S$ are bounded above. I will prove that this map is an isomorphism for all $s$ by descending induction on $s$. Assume $f_{s+1}: F_{s+1} R \rightarrow F_{s+1} S$ is an isomorphism. Consider the diagram

$$
\begin{array}{r}
0 \rightarrow F_{8} R \rightarrow F_{s+1} R \rightarrow \mathrm{Gr}_{s+1}(R) \rightarrow 0 \\
\downarrow f_{s} \quad \downarrow f_{s+1} \quad \downarrow \mathrm{Gr}_{s+1}(f) \\
0 \rightarrow F_{8} S \rightarrow F_{s+1} S \rightarrow \mathrm{Gr}_{8+1}(S) \rightarrow 0
\end{array}
$$

Since $f_{s+1}$ is an isomorphism, $\mathrm{Gr}_{s+1}(f)$ is an epimorphism. By condition (1), it must be an isomorphism. Therefore, the 5-lemma shows that $f_{s}$ is an isomorphism.

REMARK 17.1. We may dualize this by reversing the inequality (1) and 
requiring $R$ and $S$ to be bounded below. We then prove the same conclusion by ascending induction on $s$. Since $f$ is an isomorphism, $f_{s+1}$ is a monomorphism. If $f_{s}$ is an isomorphism, it follows that $\mathrm{Gr}_{s+1}(f)$ is a monomorphism. Therefore, by the reversed inequality (1), it is an isomorphism.

LeMma 17.2. Let $R$ and $S$ be filtered modules which are bounded below. Let $f: R \rightarrow S$ be a filtration preserving map which is a module isomorphism. Suppose that for every $s, \mathrm{Gr}_{s}(f): \mathrm{Gr}_{s}(R) \rightarrow \mathrm{Gr}_{s}(S)$ is an epimorphism. Then $f$ is an isomorphism of filtered modules and consequently $\operatorname{Gr}(f)$ is an isomorphism.

Proof. For small $s, f_{s}: F_{s} R \rightarrow F_{s} S$ is an isomorphism since $R$ and $S$ are bounded below. I will show that it is an isomorphism for all $s$ by ascending induction on $s$. Consider the diagram 17.1 and assume $f_{s}$ is an isomorphism. Since $f$ is an isomorphism, $f_{s+1}$ is a monomorphism. Therefore, the 5-lemma shows that $\mathrm{Gr}_{s+1}(f)$ is a monomorphism. However, it is assumed to be an epimorphism. Therefore, $\mathrm{Gr}_{s+1}(f)$ is an isomorphism and, by the 5-lemma again, so is $f_{s+1}$.

REMARK 17.2 . We may dualize Lemma 17.2 by requiring $R$ and $S$ to be bounded above and requiring each $\mathrm{Gr}_{8}(f)$ to be a monomorphism: The proof must then be replaced by that of Lemma 17.1 with minor alterations.

We now come to the comparison theorems.

TheOREM 17.1. Let $A$ and $B$ be finite dimensional chain complexes over $K \pi$. Let $B$ satisfy the conditions of Lemma 15.1. Let $f: A \rightarrow B$ be a chain map over $K \pi$ such that $f_{*}: H(A) \rightarrow H(B)$ is an isomorphism. Suppose that for all integers $r$ and $s$,

$$
\text { order } H_{s}\left(\hat{H}_{r}(\pi, A)\right) \leqq \text { order } H_{s}\left(\hat{H}_{r}(\pi, B)\right)<\infty .
$$

Then $f$ induces isomorphisms

$$
f_{* *}: H_{s}\left(\hat{H}_{r}(\pi, A)\right) \rightarrow H_{s}\left(\hat{H}_{r}(\pi, B)\right)
$$

for all $r$ and $s$.

If $K$ is a field, "order" should be replaced by "dimension."

Proof. Consider the spectral sequences defined in $\$ 15$ using the Tate complex $X$. Since $f_{*}: H(A) \rightarrow H(B)$ is an isomorphism, $f$ induces an isomorphism $I^{2}(A) \rightarrow I^{2}(B)$. Consequently, $f$ induces an isomorphism $H\left(X \otimes_{\pi} A\right)$ $\rightarrow H\left(X \otimes{ }_{\pi} B\right)$.

Now, by Lemma 15.1, $I I^{2}(B)=I I^{\infty}(B)$. Furthermore, order $I I_{r, s}^{\infty}(A)$ $\leqq$ order $I I_{r, s}^{2}(A)$ with equality holding for all $r$ and $s$ if and only if $I I^{2}(A)$ $=I I^{\infty}(A)$. But, the modules occurring in condition (1) of the theorem are just $I I_{r, s}^{2}(A)$ and $I I_{r, s}^{2}(B)$. Therefore, order $I I_{r, s}^{\infty}(A) \leqq$ order $I I_{r, s}^{\infty}(B)$ and equality can occur for all $r$ and $s$ only if $I I^{2}(A)=I I^{\infty}(A)$. Now, Lemma 17.1 applies to $f_{*}: H\left(X \otimes_{\pi} A\right) \rightarrow H\left(X \otimes_{\pi} B\right)$ using the second filtrations. Therefore, $f_{*}: I I^{\infty}(A) \rightarrow I I^{\infty}(B)$ is an isomorphism. This implies equality of orders which, 
as just observed, implies $I I^{\infty}(A)=I I^{2}(A)$. Therefore, $f_{* *}: I I^{2}(A) \rightarrow I I^{2}(B)$ is an isomorphism. This is the desired result.

Corollary 17.1. Under the conditions of Theorem 17.1, $f_{*}: H(A / \pi)$ $\rightarrow H(B / \pi)$ is an isomorphism.

This follows immediately from Theorem 17.1 and Theorem 15.1.

TheOREM 17.2. Let $A$ and $B$ be finite dimensional chain complexes over $K \pi$. Let $A$ satisfy the conditions of Lemma 15.1. Let $f: A \rightarrow B$ be a chain map over $K \pi$ such that $f_{*}: H(A) \rightarrow H(B)$ is an isomorphism. Suppose that for all integers $r$ and $s$,

$$
f^{* *}: H_{s}\left(\hat{H}_{r}(\pi, A)\right) \rightarrow H_{s}\left(\hat{H}_{r}(\pi, B)\right)
$$

is an epimorphism. Then this map is actually an isomorphism for all $r$ and $s$.

Proof. As in the proof of Theorem 17.1, $f$ induces an isomorphism $H\left(X \otimes_{\pi} A\right) \rightarrow H\left(X \otimes_{\pi} B\right)$. By Lemma 15.1, $I I^{2}(A)=I I^{\infty}(A)$. By assumption, $f_{* *}: I I^{2}(A) \rightarrow I I^{2}(B)$ is an epimorphism. Consequently, $f_{*}: I I^{\infty}(A) \rightarrow I I^{\infty}(B)$ is an epimorphism and can be an isomorphism only if $I I^{2}(B)=I I^{\infty}(B)$ and $f_{* *}: I I^{2}(A) \rightarrow I I^{2}(B)$ is an isomorphism. We now proceed exactly as in the proof of Theorem 17.1 using Lemma 17.2 in place of Lemma 17.1.

CoROllary 17.2. Under the conditions of Theorem 17.1, $f_{*}: H(A / \pi)$ $\rightarrow H(B / \pi)$ is an isomorphism.

Remark 17.2. We may dualize Theorem 17.1 by requiring $A$, not $B$, to satisfy the conditions of Lemma 15.1 and reversing the inequality (1). The proof then proceeds as in the case of Theorem 17.2.

Similarly, we may dualize Theorem 17.2 by requiring $B$, not $A$, to satisfy the conditions of Lemma 15.1 and assuming that the maps $f_{* *}$ are monomorphisms. The proof then proceeds as in the case of Theorem 17.1.

18. Proof of Theorem 6.2. As in $\$ 16$, it is sufficient to consider the case where $\pi=\pi_{p}$. Assume the theorem has been proved for all cyclic $p$-groups of order less than that of $\pi$. It is trivial if $\pi=1$. Let $\pi^{\prime}$ be the subgroup of index $p$ in $\pi$. By the inductive assumption and Remark 10.1, we can assume that

$$
\left(g / \pi^{\prime}\right)_{*}: H\left(F_{p}\left(\otimes{ }^{n} C(X)\right) / \pi^{\prime}\right) \rightarrow H\left(C\left(X^{n}\right) / \pi^{\prime}\right)
$$

is an isomorphism for all $X$. By Theorem 15.1 with $\pi / \pi^{\prime}$ in place of $\pi$, it is sufficient to prove the following lemma in order to establish Theorem 6.2.

LEMMA 18.1. The map

$$
\left(g / \pi^{\prime}\right)_{* *}: H_{s}\left(\hat{H}_{r}\left(\pi / \pi^{\prime}, F_{p}\left(\otimes^{n} C(X)\right) / \pi^{\prime}\right)\right) \rightarrow H_{s}\left(\hat{H}_{r}\left(\pi / \pi^{\prime}, C\left(X^{n}\right) / \pi^{\prime}\right)\right)
$$

is an isomorphism for all $r$ and all $X$.

Proof. There are three cases to consider. 
CASE 1. $X$ is a finite complex (i.e. $X$ has only a finite number of nondegenerate simplexes).

Let $\Delta \subset X^{n}$ be the diagonal i.e. the set of all simplexes of the form $(x, x, \cdots, x)$. Then $\Delta$ is the subcomplex of $X^{n}$ consisting of all simplexes left fixed by $\pi$. Obviously $\Delta$ and $X$ are isomorphic. The conditions of Lemma 15.1 are satisfied by $C\left(X^{n}\right) / \pi^{\prime}$ if we take $L$ to be $C(\Delta)$. Lemma 15.1 then gives

$$
H_{s}\left(\hat{H}_{r}\left(\pi / \pi^{\prime}, C\left(X^{n}\right) / \pi^{\prime}\right)\right)=H_{s}\left(\hat{H}_{r}\left(\pi / \pi^{\prime}, C(\Delta)\right)\right)=H_{s}\left(\hat{H}_{r}\left(\pi / \pi^{\prime}, C(X)\right)\right)
$$

the last inequality being an immediate consequence of the fact that $C(\Delta)$ and $C(X)$ are isomorphic. Now, Lemma 16.2 shows that all the conditions of Theorem 17.1 are satisfied. This theorem then yields the desired result.

CASE 2. $X$ is finite dimensional (i.e. for large $n, X_{n}$ contains only degenerate simplexes).

By Remark 16.2, $F\left(\otimes^{n} C(X)\right) / \pi^{\prime}$ satisfies the conditions of Lemma 15.1. By Theorem 17.2, it is sufficient to show that

$$
\left(g / \pi^{\prime}\right)_{* *}: H_{s}\left(\hat{H}_{r}\left(\pi / \pi^{\prime}, F_{p}\left(\otimes^{n} C(X)\right) / \pi^{\prime}\right)\right) \rightarrow H_{s}\left(\hat{H}_{r}\left(\pi / \pi^{\prime}, C\left(X^{n}\right) / \pi^{\prime}\right)\right)
$$

is an epimorphism for all $r$ and $s$. Now, any element $w$ of $H_{s}\left(\hat{H}_{r}\left(\pi / \pi^{\prime}, C\left(X^{n}\right) / \pi^{\prime}\right)\right)$ will have a representative cycle in $W \otimes_{\pi / \pi^{\prime}}\left(C\left(X^{n}\right) / \pi^{\prime}\right)$ where $W$ is a free acyclic complex over $\pi / \pi^{\prime}$. This representative cycle will involve only a finite number of basis elements. Therefore there is a finite subcomplex $Y \subset X$ and an element $v$ in $H_{s}\left(\hat{H}_{r}\left(\pi / \pi^{\prime}, C\left(Y^{n}\right) / \pi^{\prime}\right)\right)$ such that $v$ maps onto $w$ under the map induced by inclusion. Now, the map $\left(g / \pi^{\prime}\right)_{* *}$ for $Y$ is an isomorphism by Case 1 .

Consequently, we may take a preimage $u$ of $v$ in

$$
H_{s}\left(\hat{H}_{r}\left(\pi / \pi^{\prime}, F_{p}\left(\otimes^{n} C(Y)\right) / \pi^{\prime}\right)\right) .
$$

Because of the naturality of $g, u$ maps onto an element of

$$
\hat{H}_{s}\left(\hat{H}_{r}\left(\pi / \pi^{\prime}, F_{p}\left(\otimes^{n} C(X)\right) / \pi^{\prime}\right)\right)
$$

which in turn maps onto $w$. Since $w$ was arbitrary, this shows that $\left(g / \pi^{\prime}\right)_{* *}$ is an epimorphism and therefore, by Theorem 17.2, an isomorphism.

CASE 3. The general case. Let $r$ and $s$ be fixed. Let $Y$ be the $n(s+1)$ skeleton of $X$. By Remark 10.2, we may choose $F_{p}\left(\otimes^{n} C(X)\right)$ and $F_{p}\left(\otimes^{n} C(Y)\right)$ to be the same in dimensions $\leqq s+1$ and choose the maps $g$ for $X$ and $Y$ to agree in these dimensions. By Case 2, the map $\left(g / \pi^{\prime}\right)_{* *}$ is an isomorphism for $Y$. Consequently, it is also an isomorphism for $X$ (for the given $s$ ) since the maps are the same in dimensions $\leqq s$.

This concludes the proof of Lemma 18.1 and so of Theorem 6.2.

19. Some natural maps. In this section, I will construct some natural maps from the cohomology of $X$ to the cohomology of $C P^{n} X$. These maps generalize the cascades of Stein [23]. They lead to a natural formula for the cohomology ring of $C P^{n} X$ in terms of that of $X$ in case $K$ is a field and $H(X)$ is finitely generated in each dimension. It is quite easy to do the same thing for homology. This will be left to the reader. 
If $u \in H^{m}(X)$, we can choose a representative cocycle $u^{\prime} \in C^{m}(X)$ $=\operatorname{Hom}\left(C_{m}(X), K\right)$. Thus $u^{\prime}: C_{m}(X) \rightarrow K$ and $u^{\prime} \partial=0$. Let $M$ be a chain complex having a single generator $e$ in dimension $m$ (and so zero in all other dimensions). We may regard $u^{\prime}$ as a chain map $u^{\prime}: C(X) \rightarrow M$. If the choice of $u^{\prime}$ in the cohomology class $u$ is altered, the resulting chain maps will be homotopic. If we take a finite number of cohomology classes $u_{i} \in H^{m_{i}}(X)$, we get a chain map $\sum u_{i}^{\prime}: C(X) \rightarrow \sum M_{i}$ where $M_{i}$ has a single generator $e_{i}$ in dimension $m_{i}$. This map is again unique up to homotopy. This map is also natural up to homotopy. In other words, if $f: X \rightarrow Y$ and $u_{i}=f^{*}\left(v_{i}\right)$, then $\sum u_{i}^{\prime}: C(X)$ $\rightarrow \sum M_{i}$ and $\sum v_{i}^{\prime} f_{\sharp}: C(X) \rightarrow \sum M_{i}$ are homotopic.

Taking tensor products, applying $F$, reducing by $\pi$ and taking cohomology now gives us a map

$$
g\left(u_{1}, \cdots, u_{r}\right): H^{*}\left(F\left(\otimes^{n} \Sigma M_{i}\right) / \pi\right) \rightarrow H^{*}\left(C P^{n} X\right) .
$$

This map is natural because of Theorem 13.1 and the naturality of $\sum u_{i}^{\prime}$. The natural maps mentioned at the beginning of this section can now be defined by choosing elements $\zeta$ in $H^{*}\left(F\left(\otimes^{n} \sum M_{i}\right) / \pi\right)$ and considering the maps sending $u_{1}, \cdots, u_{r}$ to $g\left(u_{1}, \cdots, u_{r}\right) \cdot(\zeta)$. A similar construction occurs in $[22]$.

In order to define these elements $\zeta$, we consider a map of $\otimes^{n} \sum M_{i}$ onto a simpler complex in the category $\odot$. The only cases of interest are those in which $r \mid n$.

Definition 19.1. For each $r \mid n$ and each integer $d$ divisible by $n / r$, define the complex $P_{r}^{d}$ to have a single generator $a_{r}^{d}$ in dimension $d$ with relation $T^{r} a_{r}^{d}=(-1)^{d-d r / n} a_{r}^{d}$. In other dimensions, $P_{r}^{d}$ is to be zero. The sign in this definition is determined by requiring that $P_{r}^{d} \in \mathcal{P}$ (cf. Definition 6.1 (b)).

Now, suppose $u_{i} \in H^{m_{i}}(X)$ for $i=1, \cdots, r$ where $r \mid n$. Define $d=(n / r)\left(m_{1}+\cdots+m_{r}\right)$. The complex $\otimes^{n} \sum M_{i}$ has a canonical base given by the products of the $e_{i}$. Define a map $\otimes^{n} \sum M_{i} \rightarrow P_{r}^{d}$ by sending $T^{k} \otimes{ }^{n / r}\left(e_{1} \otimes \cdots \otimes e_{r}\right)$ to $T^{k} a_{r}^{d}$ and sending the remaining basis elements to zero. This, combined with the map previously considered, gives a map

$$
f_{r}\left(u_{1}, \cdots, u_{r}\right): \otimes^{n} C(X) \rightarrow P_{r}^{d} .
$$

This map, of course, depends on the choice of representative cocycles for the $u_{i}$. However, the previous results about $g\left(u_{1}, \cdots, u_{r}\right)$ show that the induced maps

$$
f_{r}^{*}\left(u_{1}, \cdots, u_{r}\right): H\left(F\left(P_{r}^{d}\right) / \pi\right) \rightarrow H\left(C P^{n} X\right)
$$

are uniquely determined by $u_{1}, \cdots, u_{r}$ and are natural.

In order to state some properties of the maps $f\left(u_{1}, \cdots, u_{r}\right)$, it is necessary to define some standard maps among the $P_{r}^{d}$.

Definition 19.2. Let $r^{\prime} \mid r$. Define $\eta: P_{r}^{d} \rightarrow P_{r^{\prime}}^{d}$ by $\eta\left(a_{r}^{d}\right)=a_{r^{\prime}}^{d}$. Define $\phi: P_{r^{\prime}}^{d} \rightarrow P_{r}^{d}$ by 


$$
\phi\left(\stackrel{d}{a} r^{\prime}\right)=\left(1+T^{r^{\prime}}+T^{2 r^{\prime}}+\cdots+T^{r-r^{\prime}}\right) a_{r}^{d}
$$

if $T^{r^{\prime}} a_{r^{\prime}}^{d}=a_{r^{\prime}}^{d}$, and by

$$
\phi\left(\begin{array}{c}
d \\
a_{r^{\prime}}
\end{array}\right)=\left(1-T^{r^{\prime}}+T^{2 r^{\prime}}-\cdots \pm T^{r-r^{\prime}}\right) a_{r}^{d}
$$

if $T^{r^{\prime}} a_{r^{\prime}}^{d}=-a_{r^{\prime}}^{d}$.

I will now state the properties of $f\left(u_{1}, \cdots, u_{r}\right)$. In the statement, it should be understood that the same representative cocycle is to be chosen for each occurrence of a particular cohomology class, and the sum of the representatives is to be taken as the representative of a sum.

Proposition 19.1. Let $u_{i} \in H^{m_{i}}(X)$ for $i=1, \cdots, r$ where $r \mid n$. Then

(1) $f_{r}\left(u_{r}, u_{1}, \cdots, u_{r-1}\right)=(-1)^{(d+1) m_{r}} T^{-1} f_{r}\left(u_{1}, \cdots, u_{r}\right)$.

(2) If $r^{\prime} \mid r$, then

$$
f_{r}\left(u_{1}, \cdots, u_{r^{\prime}}, u_{1}, \cdots, u_{r^{\prime}}, \cdots, u_{1}, \cdots, u_{r^{\prime}}\right)=\phi f_{r^{\prime}}\left(u_{1}, \cdots, u_{r^{\prime}}\right) .
$$

(3) $f_{r}\left(u_{1}^{(1)}+u_{1}^{(2)}, u_{2}, \cdots, u_{r}\right)=\sum \eta f_{k r}\left(u_{1}^{\left(i_{1}\right)}, u_{2}, \cdots, u_{r}, \cdots, u_{1}^{\left(i_{k}\right)}, u_{2}, \cdots, u_{r}\right)$.

The sum is taken over all $k \mid(n / r)$ and over certain sequences $\left(i_{1}, \cdots, i_{k}\right)$ where $i_{s}=1$ or 2 for each $s$. These sequences may be described as follows.

(a) Only those sequences are considered for which there is no $k^{\prime} \neq 0 \bmod k$ such that $i_{s}=i_{s+k^{\prime}}$, the indices larger than $k$ being reduced $\bmod k$.

(b) The sequences satisfying (a) fall into orbits under cyclic permutation. We choose exactly one from each orbit. (It does not matter which one we choose because of (1).)

The proof is simply a mechanical verification and so is left to the reader. In order to get corresponding results about the $f_{r}^{*}\left(u_{1}, \cdots, u_{r}\right)$, we must first compute the cohomology of $F\left(P_{r}^{d}\right) / \pi$ and the maps induced by $\eta$ and $\phi$. I will omit the computations which are perfectly straightforward, and will only give the results. It will only be necessary to use these results in the case where $K$ is a field of characteristic $p$ and $n$ is a power of $p$. Consequently, I will only state the results for this case.

PROPOSITION 19.2. Let $K$ be a field of characteristic $p$ and let $n$ be a power of p. Then, for each $i, H^{i}\left(F\left(P_{r}^{d}\right) / \pi\right)$ is either 0 or has a single generator $\zeta_{r}^{d, i} . I$ will set $\zeta_{r}^{d, i}=0$ if $H^{i}=0$. Then, if $r \neq n$, we have $\zeta_{r}^{d, t} \neq 0$ if and only if $d \geqq i \geqq(d r / n)$ +2 . If $r=n$, only $\zeta_{r}^{d, d} \neq 0$.

Proposition 19.3. Let $K, p, n, \zeta_{r}^{a, 4}$ be as in Proposition 19.2. The maps of cohomology induced by $\eta$ and $\phi$ are as follows.

$$
\eta^{*}\left(\zeta_{r^{\prime}}^{d, i}\right)=0 \text { if } d-i \text { is even and } r \neq r^{\prime}
$$

$$
\eta^{*}\left(\zeta_{r^{\prime}}^{d, i}\right)=\zeta_{r}^{d, i} \text { if } d-i \text { is odd. }
$$


(b)

$$
\begin{aligned}
& \phi^{*}\left(\zeta_{r}^{d, i}\right)=\zeta_{r^{\prime}}^{d, i} \text { if } d-i \text { is even, } \\
& \phi^{*}\left(\zeta_{r}^{d, i}\right)=0 \text { if } d-i \text { is odd and } r \neq r^{\prime} .
\end{aligned}
$$

Note that $\eta$ and $\phi$ are the identity maps if $r=r^{\prime}$.

In performing these calculations, we make use of the observation that, if $K$ has characteristic $p$, then $j_{p}: H\left(F_{p}(P) / \pi\right) \rightarrow H(F(P) / \pi)$ is an isomorphism. This is an immediate consequence of Corollary 12.1 and Theorem 11.1.

It is now possible to define natural functions $h_{r}^{i}\left(u_{1}, \cdots, u_{r}\right) \in H^{i}\left(C P^{n} X\right)$ by setting

$$
h_{r}^{i}\left(u_{1}, \cdots, u_{r}\right)=f_{r}^{*}\left(u_{1}, \cdots, u_{r}\right) \cdot\left(\zeta_{r}^{d, i}\right)
$$

where $d=(n / r)\left(m_{1}+\cdots+m_{r}\right)$ if $u_{i} \in H^{m_{i}}(X)$. The properties of these functions can be written down immediately from the preceding three propositions.

Proposition 19.4. Let $K$ be a field of characteristic $p$ and let $n$ be a power of p. Let $d$ and $m_{i}$ be as above. Then

(1) $h_{r}^{i}\left(u_{1}, \cdots, u_{r}\right)=0$ if $i>d$ or $i \leqq(d r / n)+1$. If $r=n$, this last condition should be replaced by $i<d$.

(2) $h_{r}^{i}\left(u_{r}, u_{1}, \cdots, u_{r-1}\right)=(-1)^{(d+1) m_{r}} h_{r}^{i}\left(u_{1}, \cdots, u_{r}\right)$.

(3) If $r^{\prime} \mid r$ but $r^{\prime} \neq r$, then $h_{r}^{4}\left(u_{1}, \cdots, u_{r^{\prime}}, \cdots, u_{1}, \cdots, u_{r^{\prime}}\right)$ is 0 if $d-i$ is odd and is $h_{r^{\prime}}^{i}\left(u_{1}, \cdots, u_{r^{\prime}}\right)$ if $d-i$ is even.

(4) If $d-i$ is even, $h_{r}^{i}$ is multilinear. If $d-i$ is odd, then

$$
\begin{aligned}
h_{r}^{i}\left(u_{1}^{(1)}+u_{1}^{(2)}, u_{2}, \cdots\right. & \left., u_{r}\right) \\
& =\sum h_{k r}^{i}\left(u_{1}^{\left(i_{1}\right)}, \cdots, u_{r}, u_{1}^{\left(i_{2}\right)}, \cdots, u_{r}, \cdots, u_{1}^{\left(i_{k}\right)}, \cdots, u_{r}\right)
\end{aligned}
$$

the sum being over the same $\left(i_{1}, \cdots, i_{k}\right)$ as in Proposition 19.1.

The functions $h_{r}^{4}$ have an additional property which will be used in proving Theorem 21.1.

Proposition 19.5. Let $K, p$, and $n$ be as in Proposition 19.4. Assume $H^{*}(X)$ finitely generated in each dimension. Let $v_{i}$ be a base for $H^{*}(X)$. Then a base for $H^{*}\left(C P^{n} X\right)$ is given by $h_{r}^{i}\left(v_{i_{1}}, \cdots, v_{i_{r}}\right)$ where $d \geqq i \geqq(d r / n)+2$ with $d=(n / r) \sum \operatorname{dim} v_{i_{s}}, r$ runs over all divisors of $n$, and the set of sequences $\left(i_{1}, \cdots, i_{r}\right)$ is given as follows.

(a) There is no $r^{\prime} \not \equiv 0 \bmod r$ such that $i_{s}=i_{s+r^{\prime}}$ for all $s$ (the indices $>r$ being reduced $\bmod r$ ).

(b) Consider the set of sequences satisfying (a). Then exactly one is chosen from each orbit under cyclic permutation.

Proof. Since $K$ is a field, we can find a chain map $H(X) \rightarrow C(X)$ inducing 
an isomorphism of homology. Consequently, $H^{*}\left(C P^{n} X\right)=H^{*}\left(F\left(\otimes^{n} H(X)\right) / \pi\right)$.

To complete the proof, it is merely necessary to observe that $\otimes^{n} H(X)$ splits up into a direct sum of complexes isomorphic to various $P_{r}^{d}$. The projections on the summands are given by the maps $f_{r}\left(v_{i_{1}}, \cdots, v_{i_{r}}\right)$ where $r$ and $\left(i_{1}, \cdots, i_{r}\right)$ satisfy the conditions given in the Proposition.

20. Some universal maps. We can consider functions from modules to modules satisfying conditions such as those in Proposition 19.4 as "QBmaps" and ask whether there are universal ones. I will give two such universal maps here.

Proposition 20.1. Let $K$ be a field of characteristic $p$ and let $n^{\prime}$ be a power of p. Let $H$ be a graded $K$-module. Consider the functions $\lambda_{r}\left(u_{1}, \cdots, u_{r}\right)=i m a g e$ of $\otimes^{n^{\prime} / r}\left(u_{1} \otimes \cdots \otimes u_{r}\right)$ in $\otimes^{n^{\prime}} H / \pi$, defined for all $r \mid n^{\prime}$ and all $u_{i} \in H^{m_{i}}$ such that $\left(n^{\prime} / r\right)\left(\sum m_{i}\right)=d^{\prime}$ where $d^{\prime}$ is some fixed integer. These functions are universal among functions with the following properties.

$$
\begin{gathered}
\lambda_{r}\left(u_{r}, u_{1}, \cdots, u_{r-1}\right)=(-1)^{\left(d^{\prime}+1\right) m_{r} \lambda_{r}\left(u_{1}, \cdots, u_{r}\right) .} \\
\lambda_{r}\left(u_{1}, \cdots, u_{r^{\prime}}, u_{1}, \cdots, u_{r^{\prime}}, \cdots, u_{1}, \cdots, u_{r^{\prime}}\right)=\lambda_{r^{\prime}}\left(u_{1}, \cdots, u_{r^{\prime}}\right) .
\end{gathered}
$$

$\lambda_{r}$ is multilinear.

The proof consists of a trivial verification.

If $\pi$ acts on a module $A$, I will denote by $A^{*}$ the set of elements fixed un$\operatorname{der} \pi$.

Proposition 20.2. Let $K, p, n^{\prime}$, and $H$ be as in Proposition 20.1. Consider the functions

$$
\gamma_{r}\left(u_{1}, \cdots, u_{r}\right)=\left(1+T+\cdots+T^{r-1}\right)\left(\otimes^{n^{\prime} / r}\left(u_{1} \otimes \cdots \otimes u_{r}\right)\right)
$$

in $\left(\otimes n^{\prime} H\right)^{\pi}$, defined for all $r \mid n^{\prime}$ and all $u_{i} \in H^{m_{i}}$ such that $\left(n^{\prime} / r\right)\left(\sum m_{i}\right)=d^{\prime}$ where $d^{\prime}$ is some fixed integer. These functions are universal among those functions having the following properties.

$$
\begin{aligned}
& \gamma_{r}\left(u_{r}, u_{1}, \cdots, u_{r-1}\right)=(-1)^{\left(d^{\prime}+1\right) m_{r}} \gamma_{r}\left(u_{1}, \cdots, u_{r}\right), \\
& \gamma_{r}\left(u_{1}, \cdots, u_{r^{\prime}}, \cdots, u_{1}, \cdots, u_{r^{\prime}}\right)=0 \text { if } r^{\prime} \neq r, \\
& \gamma_{r}\left(u_{1}^{(1)}+u_{1}^{(2)}, u_{2}, \cdots, u_{r}\right)=\sum \gamma_{k r}\left(u_{1}^{\left(i_{1}\right)}, \cdots, u_{r}, \cdots, u_{1}^{\left(i_{k}\right)}, \cdots, u_{r}\right),
\end{aligned}
$$

the sum being over the same $\left(i_{1}, \cdots, i_{k}\right)$ as in Proposition 19.1.

The proof again is simply a mechanical verification.

Now, consider the maps $h_{r}^{i}$ defined in $\$ 19$. If $d=(n / r)\left(\sum \operatorname{dim} u_{i}\right)$, then $h_{r}^{i}$ is zero for $r=n$ if $p(i-2) \geqq d \geqq i$, and it is zero for $r \geqq n / p^{s}$ for $p^{s+1}(i-2) \geqq d$ $\geqq p^{s}(i-2)$. We can therefore apply Propositions 20.1 and 20.2 with $d^{\prime}=d / p$, resp. $d / p^{\circ+1}$ in the two cases just mentioned. This leads immediately to a formula for $H\left(C P^{n} X\right)$. 
21. A formula. In this section, I will no longer assume that $n$ is a power of $p$. For each power $p^{k}$ of $p$ which divides $n$, define $\pi\left(p^{k}\right)$ to be the subgroup of $\pi$ of index $p^{k}$.

Let $K$ be a field of characteristic $p$ and let $H=H^{*}(X, K)$. If $A$ is any graded module, let $[A]^{k}$ be the $k$-dimensional part of $A$.

Definition 21.1. If $p^{r} \mid n$ and $j$ and $k$ are any integers, define $G_{k, n, r^{r}}^{j}$ to be $\left[\left(\otimes^{n / p^{r}} H\right) / \pi\left(p^{r}\right)\right]^{k}$ if $p^{r} k-j$ is even, and define it to be $\left[\left(\otimes^{n / p^{r}} H\right)^{\pi\left(p^{r}\right)}\right]^{k}$ if $p^{r} k-j$ is odd. We regard $G$ as having dimension $j$ not $k$ in considering $G$ as a graded module.

Definition 21.2. For all $j$, define

$$
J^{j}=\sum_{j-2 \geqq k>j / p} G_{k, n, p}^{j}+\sum_{r \geqq 2} \sum_{j-2 \geqq k \geqq(j-1) / p} G_{k, n, p^{r}}^{j} .
$$

Theorem 21.1. Let $K$ have characteristic p. Assume $H(X)$ finitely generated in each dimension. Then there are natural isomorphisms

$$
\begin{array}{ll}
H^{j}\left(C P^{n} X\right) \approx\left[\left(\otimes^{n} H\right) / \pi\right]^{j}+J^{i} & \text { for } j>0, \\
H^{0}\left(C P^{n} X\right) \approx\left(\otimes^{n} H^{0}\right)^{\pi} &
\end{array}
$$

where $H=H(X, K)$.

Proof. If $n$ is a power of $p$, we map the right hand side into $H\left(C P^{n} X\right)$ as described at the end of $\S 20$. This map is clearly natural. To prove it is an isomorphism, we merely choose a base for $H$ and compare the resulting base for the right hand side with the one given for $H\left(C P^{n} X\right)$ by Proposition 19.5.

Now, let $n$ be arbitrary and let $\pi_{p}$ be the Sylow $p$-group of $\pi$. Let $p^{h}$ be the largest power of $p$ dividing $n$. Let $n^{\prime}=n / p^{h}$. Then

$$
C P^{n} X=C P^{p^{h}}\left(X^{n^{\prime}}\right) /\left(\pi / \pi_{p}\right) \quad \text { where } \pi / \pi_{p},
$$

which is cyclic of order $n^{\prime}$, acts on $C P^{p^{h}}\left(X^{n^{\prime}}\right)$ by acting in the usual way on $X^{n^{\prime}}$. By Theorem 11.2, dualized to cohomology,

$$
H\left(C P^{n} X\right) \approx H\left(C P^{p h}\left(X^{n^{\prime}}\right)\right)^{\pi / \pi_{p}} .
$$

We compute $H\left(C P^{p^{h}}\left(X^{n^{\prime}}\right)\right)$ by Theorem 21.3 which has just been proved for powers of $p$. The result then follows immediately from the following well known lemma.

Lemma 21.1. Let $G$ be a group of order prime to $p$ acting on a $K$-module $A$ where $K$ is a field of characteristic $p$. Then $A^{G} \approx A / G$ naturally.

Proof. Consider the maps $\eta$ and $\phi$ defined in $\S 11$. We have $\eta \mid A^{G}: A^{G} \rightarrow A / G$ and $\phi: A / G \rightarrow A^{G}$. The compositions are just multiplication by the order of $G$ and so are isomorphisms.

I will now give some further properties of the formula given by Theorem 21.1.

The quotient map $f: X^{n} \rightarrow C P^{n} X$ induces a map $f^{*}$ of cohomology. 
TheOREM 21.2. For all $j, f^{*}\left(J^{j}\right)=0$. If $a \in\left(\otimes^{n} H\right) / \pi$ is represented by $a^{\prime} \in \otimes^{n} H$, then $f^{*}(a)=\sigma a^{\prime}$ where $\sigma=\sum_{t \in \pi} t$. In dimension $0, f^{*}$ is simply the inclusion $\left(\otimes^{n} H^{0}\right)^{\pi} \rightarrow \otimes^{n} H^{0}$.

To prove this, we notice that

$$
u / \pi:\left(\otimes^{n} H\right) / \pi \rightarrow F\left(\otimes^{n} H\right) / \pi
$$

induces $f^{*}$ in cohomology. We then simply choose a basis for $H$ and see what $u / \pi$ does to basis elements. I will omit all details.

TheOREM 21.3. The cup products in $H^{*}\left(C P^{n} X\right)$ are given as follows.

(1) Let $a$ and $b$ have dimension $>0$. Then,

(i) If $a$ or $b$ is in $J^{i}$, then $a b=0$.

(ii) Let $a, b \in\left(\otimes^{n} H\right) / \pi$ be represented by $a^{\prime}, b^{\prime} \in \otimes^{n} H$. Then $a b$ is represented by $\left(\sigma a^{\prime}\right) b^{\prime}$ and also by $a^{\prime}\left(\sigma b^{\prime}\right)$ where $\sigma$ is as in Theorem 21.2.

(2) Let $a \in\left(\otimes^{n} H^{0}\right)^{x}$. Let $b$ belong to any one of the summands in the formula for $H^{*}\left(C P^{n} X\right)$, say one of the form $\left(\otimes^{n / p^{r}} H\right) / \pi$ or $\left(\otimes^{n / p^{r}} H\right)^{\pi}$. Then $a b=d^{*} a \cdot b$ where the second product is the usual one in $\otimes^{n / p^{r}} H$ and $d^{*}: \otimes^{n} H^{0}$ $\rightarrow \otimes^{n / p^{r}} H^{0}$ is induced by the diagonal map $d: X^{n / p^{r}} \rightarrow X^{n}$.

REMARK 21.1. This map $d^{*}$ is completely determined by $H^{0}$ itself. $H^{0}$ has a base of mutually orthogonal idempotents $e_{i}$, one for each component of $X$. This base is determined up to permutation by the multiplicative structure of $H^{0}$. Now,

$$
d^{*}\left(\otimes^{p r}\left(e_{i_{1}} \otimes \cdots \otimes e_{i_{n / p} r}\right)\right)=e_{i_{1}} \otimes \cdots \otimes e_{i_{n} / p^{r}}
$$

while $d^{*}$ annihilates the remaining elements in the canonical base for $\otimes^{n} H^{0}$ (i.e. the base consisting of products of the $e_{i}$ ).

I will now outline the proof of Theorem 21.3, again omitting the details of the computation. As in the proof of Theorem 21.1, it will again be sufficient to consider only the case where $n$ is a power of $p$. Choose a basis for $H_{*}$ $=H_{*}(X, K)$. This gives a canonical decomposition of $\otimes^{n} H_{*}$ into a direct sum of $P_{r}^{d}$ as in the proof of Proposition 19.5, and $H^{*}\left(C P^{n} X\right)$ is a direct sum of the $H^{*}\left(F\left(P_{r}^{d}\right) / \pi\right)$. By the proof of Theorem 21.1, a canonical base for $H^{*}\left(C P^{n} X\right)$ is given by the images of the canonical basis elements of the $H^{*}\left(F\left(P_{r}^{d}\right) / \pi\right)$. To check that two maps of $\otimes^{n} H$ induce the same maps on $H^{*}\left(F\left(\otimes^{n} H\right) / \pi\right)$, it is sufficient to check this for their compositions with the canonical injections induced by the inclusions $P_{r}^{d} \rightarrow \otimes^{n} H$.

To prove the formulas for cup products, it is sufficient to check them on basis elements. Let $d: H_{*} \rightarrow H_{*} \otimes H_{*}$ be the diagonal map. Consider the maps

$$
P_{r}^{d} \rightarrow \otimes^{n} H_{*} \stackrel{d}{\rightarrow} \otimes{ }^{n} H_{*} \otimes \otimes{ }^{n} H_{*} \rightarrow P_{r^{\prime}}^{d^{\prime}} \otimes P_{r^{\prime \prime}}^{d^{\prime \prime}}
$$

If we compute all the induced maps

$$
H^{*}\left(F\left(P_{r^{\prime}}^{d^{\prime}}\right) / \pi\right) \otimes H^{*}\left(F\left(P_{r^{\prime \prime}}^{d^{\prime \prime}}\right) / \pi\right) \rightarrow H^{*}\left(F\left(P_{r}^{d}\right) / \pi\right)
$$


we will then know the cup products of all the basis elements. In case $r^{\prime}=r^{\prime \prime}=n$ or if $d^{\prime}$ or $d^{\prime \prime}$ is 0 , this is essentially trivial. In the remaining cases, we use the following lemma.

Lemma 21.1. Assume $d^{\prime}, d^{\prime \prime}>0$ and not both $r^{\prime}$ and $r^{\prime \prime}$ are equal to $n$. Then any map $P_{r}^{d} \rightarrow P_{r}^{d^{\prime}} \otimes P_{r}^{d_{\prime \prime \prime}^{\prime \prime}}$ induces the zero map

$$
H^{*}\left(F\left(P_{r^{\prime}}^{d^{\prime}}\right) / \pi\right) \otimes H^{*}\left(F\left(P_{r^{\prime \prime}}^{d^{\prime \prime}}\right) / \pi\right) \rightarrow H^{*}\left(F\left(P_{r}^{d}\right) / \pi\right) .
$$

Proof. We can clearly assume $d=d^{\prime}+d^{\prime \prime}$. Let $r_{0}$ be the larger of $r^{\prime}$ and $r^{\prime \prime}$. Then any map $P_{r}^{d} \rightarrow P_{r}^{d^{\prime}} \otimes P_{r}^{d^{\prime \prime \prime}}$ factors through the left universal map

$$
\psi: P_{r_{0}}^{d} \rightarrow P_{r^{\prime}}^{d^{\prime}} \otimes P_{r^{\prime \prime}}^{d^{\prime \prime}}
$$

given by $\psi\left(a_{r_{0}}^{d}\right)=a_{r}^{d,} \otimes a_{r^{\prime \prime}}^{d^{\prime \prime}}$. It is therefore sufficient to verify the lemma for $\psi$, which is done by direct computation.

22. Computations when $K=Z$. Any general formula for the case $K=Z$ would appear to be extremely complicated. I will indicate here how to make the computation in the case where $n$ is a prime $p$. I will not give the explicit formulas since these agree with those found by Yoshioka [27] for finite complexes by a completely different method. The method given here is itself interesting, however, since it can be used to prove a lemma used by Dyer and Lashof [7] in their theory of homology operations.

In order to compute $F\left(\otimes^{n} M\right) / \pi$ for any $Z$-free chain complex $M$, we split $M$ into a direct sum of elementary chain complexes as in the proof of Lemma 16.2. The cross product terms $P$ in $\otimes^{n} M$ will be $K \pi$-free and so we can take $F(P)=P$. This, of course, uses the fact that $n$ is prime. Because of this, it is sufficient to find $F\left(\otimes^{n} M\right) / \pi$ when $M$ is elementary. If $M$ is of type 1 , this is trivial. Therefore, suppose $M$ is of type 2 with two generators $a$ and $b$ of dimensions $m+1$ and $m$ with $\partial a=\theta b$. If $p \nmid \theta$, we apply the following lemma.

LEMma 22.1. Let $P \in \odot$ be such that $H(P)$ consists entirely of torsion elements of order prime to $p$. Let the order of $\pi$ be a power of $p$. Then $H(F(P) / \pi)$ is isomorphic to the subgroup of $H(P / \pi)$ consisting of torsion elements of order prime to $p$.

Proof. Let $\mathcal{C}$ be the Serre class of torsion groups with no $p$-torsion. By Lemma 16.1, $H(F(P) / \pi) \in \mathcal{C}$. The result now follows from Theorem 11.2 applied to $P$ and to $F(P)$ because $H(P)=H(F(P))$.

Now, consider the case where $p \mid \theta$. The only nontrivial partial $p$-resolutions in $F\left(\otimes^{p} M\right)$ will be $W^{(p m)}$ and $W^{(p m+p)}$. It is necessary to choose the attaching map $\psi$ for $W^{(p m+p)}$ in a special way. Let $M^{\prime}$ be the same as $M$ as a module but with a new boundary operator $\partial^{\prime} a=b$. Attach $W^{(m p)}$ to $\otimes^{p} M^{\prime}$ and define the map $\psi^{\prime}$ attaching $W^{(p m+p)}$ to the resulting complex. Now, let $\psi_{k}$ be $\theta^{m p+p+1-k} \psi_{k}^{\prime}$ in dimensions $k$ such that $m p+1 \leqq k \leqq m p+p$ and let $\psi_{k}$ be $\theta^{p} \psi_{k}^{\prime}$ in dimensions $k \leqq m p$. Now this is a map attaching $W^{(p m+p)}$ to $\left(\otimes^{p} M\right)$ 
$\cup W^{(p m)}$ and contains a factor $\theta$. Because of this, it is very easy to compute the part of the homology $H\left(F\left(\otimes^{n} M\right) / \pi\right)$ which is due to the presence of $W^{(m p)}$ and $W^{(m p+p)}$. Thus, in this case, the problem again reduces to that of computing $H\left(\otimes^{p} M / \pi\right)$. This is trivial for $p=2$ so I will assume $p$ is odd.

The main step is the calculation of $H\left(\otimes^{p} M^{\prime} / \pi\right)$ where $M^{\prime}$ is the complex defined above.

Lemma 22.2. Let $X$ be the $K \pi$-complex given by the following sequence

$$
0 \rightarrow K \stackrel{\sigma}{\rightarrow} K \pi \stackrel{\tau}{\rightarrow} K \pi \stackrel{\sigma}{\rightarrow} \cdots \stackrel{\tau}{\rightarrow} K \pi \stackrel{1}{\rightarrow} K \rightarrow 0
$$

where $X_{i}=K$ for $i=m p$ and $m p+p, K \pi$ for $m p<i<m p+p$ and is zero otherwise. Here, $\tau=T-1$ and $\sigma=1+T+T^{2}+\cdots+T^{p-1}$.

Then there is a homotopy equivalence $\psi: X \rightarrow \otimes^{p} M^{\prime}$ such that $\psi_{m p}(1)=\otimes^{p} b$ and $\psi_{m p+p}(1)=\gamma\left(\otimes^{p} a\right)$ with $\gamma \neq \equiv 0 \bmod p$.

Proof. $\otimes^{p} M^{\prime}$ is isomorphic to $K$ in dimensions $m p$ and $m p+p$ and is $K \pi$-free in the other dimensions. Also, $H\left(\otimes^{p} M^{\prime}\right)=0$. Consequently, by using the usual free to acyclic mapping theorems, we can construct $\psi$, a $K \pi$-map $\otimes{ }^{p} M^{\prime} \rightarrow X$, and homotopies between the compositions and the corresponding identity maps.

To show $\gamma \not \equiv 0 \bmod p$, we simply note that $H_{m p+p}\left((X / \pi) \otimes Z_{p}\right)=Z_{p}$. Since $\psi$ is a homotopy equivalence, it cannot induce the zero map in dimension $m p+p$.

Now, by using this lemma, we can immediately compute $H\left(\otimes^{p} M^{\prime} / \pi\right)$. We can split $\otimes^{p} M^{\prime} / \pi$ into a direct sum of elementary complexes. The nontrivial ones (i.e. ones with nonzero homology) can be determined from $H\left(\otimes^{p} M^{\prime} / \pi\right)$. The number of trivial ones can then be found by counting the number of basis elements of $\otimes^{p} M^{\prime} / \pi$. Now, $\otimes^{p} M / \pi$ is obtained from $\otimes^{p} M^{\prime} / \pi$ by multiplying each boundary operator by $\theta$. This gives us immediately a decomposition of $\otimes^{p} M / \pi$ into elementary complexes from which we compute $H\left(\otimes^{p} M / \pi\right)$.

REMARK 22.1. It can be shown (by constructing an explicit map $\psi$ ) that $\gamma \equiv(-1)^{m(p-1) / 2}((p-1) / 2) ! \bmod p$ and that any integral representative of this can be realized by an appropriate $\psi$.

\section{BIBLIOGRAPHY}

1. N. Bourbaki, Algèbre multilinéaire, Part 1, Book 2, Paris, Hermann et Cie, Chapter 3, Appendix III.

2. H. Cartan and S. Eilenberg, Homological algebra, Princeton, 1956.

3. H. Cartan, Séminaire E.N.S., 1950-1951.

4. - Séminaire E.N.S. 1954-1955.

5. P. E. Conner, Concerning the action of a finite group, Proc. Nat. Acad. Sci. U.S.A. vol. 42 (1956) pp. 349-351.

6. A. Dold, Homology of symmetric products and other functors of complexes, Ann. of Math. vol. 68 (1958) pp. 54-80. 
7. E. Dyer and R. Lashof, Homology of iterated loop spaces, to appear.

8. B. Eckmann, Cohomology of groups and transfer, Ann. of Math. vol. 58 (1953) pp. 481493.

9. - Coverings and Betti numbers, Bull. Amer. Math. Soc. vol. 55 (1949) pp. 95-101.

10. S. Eilenberg and S. MacLane, On the groups $H(\pi, n)$. II, Ann. of Math. vol. 60 (1954) pp. 49-139.

11. S. Eilenberg and N. Steenrod, Foundations of algebraic topology, Princeton, 1952.

12. G. Hochschild, Relative homological algebra, Trans. Amer. Math. Soc. vol. 82 (1956) pp. 246-269.

13. D. M. Kan, Adjoint functors, Trans. Amer. Math. Soc. vol. 87 (1958) pp. 294-329.

14. J. Milnor, The geometric realization of a semi-simplicial complex, Ann. of Math. vol. 65 (1957) pp. 357-362.

15. J. C. Moore, Notes on semi-simplicial complexes (mimeographed), Princeton, 1956.

16. M. Nakaoka, Cohomology theory of a complex with a transformation of prime period and its applications, J. Inst. Polytech. Osaka City Univ. Ser. A vol. 7 (1956) pp. 51-102.

17. M. Richardson, On the homology characters of symmetric products, Duke Math. J. vol. 1 (1935) pp. 50-69.

18. M. Richardson and P. A. Smith, Periodic transformations of complexes, Ann. of Math. vol. 39 (1938) pp. 611-633.

19. P. Samuel, Universal mappings and free topological groups, Bull. Amer. Math. Soc. vol. 54 (1948) pp. 591-598.

20. J-P. Serre, Groupes d'homotopie et classes de groupes abéliens, Ann. of Math. vol. 58 (1953) pp. 258-294.

21. P. A. Smith, Fixed point theorems for periodic transformations, Amer. J. Math. vol. 63 (1941) pp. 1-8.

22. N. Steenrod, Cohomology operations derived from the symmetric group, Comment. Math. Helv. vol. 31 (1957) pp. 195-217.

23. S. K. Stein, Homology of the two fold symmetric product, Ann. of Math. vol. 59 (1954) pp. 570-583.

24. R. G. Swan, $A$ new method in fixed point theory, Comment. Math. Helv. vol. 34 (1960) pp. 1-16.

25. - Homology of cyclic products (mimeographed), Reports, Seminar in Topology, University of Chicago, Summer, 1957.

26. - On the cochains of orbit spaces, to appear.

27. T. Yoshioka, Base canonique d'homologie du produit d'ordre p d'un complexe fini ( $p$ premier impair), Osaka Math. J. vol. 10 (1958) pp. 11-29.

University of Chicago, Chicago, Illinois 